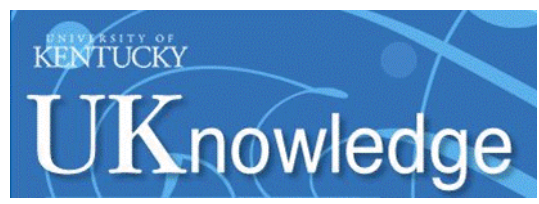

University of Kentucky

UKnowledge

$12-1-2015$

\title{
Evidence for Photoionization-Driven Broad Absorption Line Variability
}

Tinggui Wang

University of Science and Technology of China, China

Chenwei Yang

University of Science and Technology of China, China

Huiyuan Wang

University of Science and Technology of China, China

Gary J. Ferland

University of Kentucky, gary@uky.edu

Follow this and additional works at: https://uknowledge.uky.edu/physastron_facpub

Part of the Astrophysics and Astronomy Commons, and the Physics Commons

Right click to open a feedback form in a new tab to let us know how this document benefits you.

\section{Repository Citation}

Wang, Tinggui; Yang, Chenwei; Wang, Huiyuan; and Ferland, Gary J., "Evidence for Photoionization-Driven Broad Absorption Line Variability" (2015). Physics and Astronomy Faculty Publications. 368.

https://uknowledge.uky.edu/physastron_facpub/368

This Article is brought to you for free and open access by the Physics and Astronomy at UKnowledge. It has been accepted for inclusion in Physics and Astronomy Faculty Publications by an authorized administrator of UKnowledge. For more information, please contact UKnowledge@lsv.uky.edu. 


\section{Evidence for Photoionization-Driven Broad Absorption Line Variability}

Digital Object Identifier (DOI)

https://doi.org/10.1088/0004-637X/814/2/150

\section{Notes/Citation Information}

Published in The Astrophysical Journal, v. 814, no. 2, article 150, p. 1-17.

(c) 2015. The American Astronomical Society. All rights reserved.

The copyright holders have granted the permission for posting the article here. 


\title{
EVIDENCE FOR PHOTOIONIZATION-DRIVEN BROAD ABSORPTION LINE VARIABILITY
}

\author{
Tinggui Wang ${ }^{1}$, Chenwei Yang $^{1}$, Huiyuan $\mathrm{WANG}^{1}$, and Gary Ferland ${ }^{2,3}$ \\ ${ }^{1}$ CAS Key Laboratory for Research in Galaxies and Cosmology, Department of Astronomy, University of Science and Technology of China, \\ Hefei, Anhui 230026, China; twang@ustc.edu.cn \\ ${ }^{2}$ Department of Physics and Astronomy, University of Kentucky, Lexington, KY 40506, USA \\ ${ }^{3}$ Centre for Theoretical Atomic, Molecular and Optical Physics, School of Mathematics and Physics, Queens University Belfast, Belfast BT7 1NN, UK \\ Received 2014 November 7; accepted 2015 September 8; published 2015 December 1
}

\begin{abstract}
We present a qualitative analysis of the variability of quasar broad absorption lines using the large multi-epoch spectroscopic data set of the Sloan Digital Sky Survey Data Release 10. We confirm that variations of absorption lines are highly coordinated among different components of the same ion or the same absorption component of different ions for $\mathrm{C}$ IV, Si IV, and N v. Furthermore, we show that the equivalent widths (EWs) of the lines decrease or increase statistically when the continuum brightens or dims. This is further supported by the synchronized variations of emission and absorption-line EWs when the well-established intrinsic Baldwin effect for emission lines is taken into account. We find that the emergence of an absorption component is usually accompanied by the dimming of the continuum while the disappearance of an absorption-line component is accompanied by the brightening of the continuum. This suggests that the emergence or disappearance of a $\mathrm{C}$ IV absorption component is only the extreme case, when the ionic column density is very sensitive to continuum variations or the continuum variability the amplitude is larger. These results support the idea that absorption-line variability is driven mainly by changes in the gas ionization in response to continuum variations, that the line-absorbing gas is highly ionized, and in some extreme cases, too highly ionized to be detected in UV absorption lines. Due to uncertainties in the spectroscopic flux calibration, we cannot quantify the fraction of quasars with asynchronized continuum and absorption-line variations.
\end{abstract}

Key words: line: formation - quasars: absorption lines - quasars: emission lines

Supporting material: machine-readable table

\section{INTRODUCTION}

It is generally accepted that quasar feedback plays a crucial role in the context of galaxy formation and evolution. The bimodal color distributions of galaxies in the present universe and the deficiency of massive galaxies in the mass function of galaxies in comparison with dark-matter halo mass distributions require quenching of massive galaxies (Silk 2011). The correlations between masses of supermassive black holes (SMBHs) and the bulge of their host galaxies suggest that the growth of galaxies and SMBHs at their centers are closely connected (e.g., Kormendy \& Ho 2013). Considering the high radiation efficiency of black hole accretion, even if a small fraction of the enormous power from accreting SMBHs during the quasar phase goes into the interstellar medium in the host galaxy, it can interrupt the growth of both the black hole and its host galaxy. Quasar outflow is a natural form of such feedback, given its ubiquity among quasars, with a potentially large kinetic power, as demonstrated in a few quasars with UV broad absorption lines (BALs) from $\mathrm{S}_{\text {IV }}, \mathrm{O}_{\text {IV }}$, or $\mathrm{Fe}_{\text {II }}$ excited states or ionized X-ray absorption lines (e.g., Korista et al. 2008; Chartas et al. 2009; Arav et al. 2013; Borguet et al. 2013; Tombesi et al. 2015). Outflows are manifested as blueshifted broad emission lines (BELs) or BALs, imprinted on UV or $\mathrm{X}$-ray spectra of quasars. However, the total kinetic energy of outflows on these scales, as well as the way they interact with the ISM, remains very uncertain because of the uncertainty in the geometry and the total column density of quasar absorbers, together with the complication of more than one stable phase (Arav et al. 2013).

Quasar absorption lines were divided into two classes according to their origins. The intervening absorption lines, which are produced by galaxies and intergalactic material between the quasar and the observer, are not the subject of this study. Intrinsic absorption lines, formed by gas either directly associated with the quasar or in its host galaxy, are our main focus. Most intrinsic absorption lines are blueshifted with respect to the corresponding emission lines, implying that the gas is flowing out of the center. Intrinsic absorption lines are further classified into narrow and broad absorption lines (NALs and BALs) according to the width of the absorption line (e.g., Weymann et al. 1991; Barlow \& Sargent 1997; Barlow et al. 1997). In this paper, we will focus on BALs, but we do not distinguish BALs and mini-BALs with a width of $500-2000 \mathrm{~km} \mathrm{~s}^{-1}$ (Hamann \& Sabra 2004) as there is a continuous distribution of absorption index between the two classes (e.g., Trump et al. 2006; Ganguly \& Brotherton 2008; Gibson et al. 2009) and there are instances where a source can transition from mini-BALs to BALs (e.g., Rodríguez Hidalgo et al. 2013). It has long been known that BALs are variable on timescales from years to months (Foltz et al. 1987; Smith \& Penston 1988; Barlow et al. 1989, 1992; Barlow 1993; Turnshek et al. 1998), but systematic studies have only recently been carried out (Lundgren et al. 2007; Gibson et al. 2008, 2010; Capellupo et al. 2011, 2012, 2014; Filiz Ak et al. 2012, 2013; Welling et al. 2014).

Capellupo et al. $(2011,2014)$ monitored a sample of $24 \mathrm{BAL}$ QSOs at $z \sim 2$ over timescales of 0.02 to 8.7 years ${ }^{4}$ and found that $65 \%$ of their quasars displayed BAL variability over several years and $39 \%$ on timescales of less than one year.

\footnotetext{
4 Time will be referred in rest frame of quasars unless explicitly state otherwise.
} 
Similarly, Wildy et al. (2014) analyzed 2-epoch spectra of 50 BAL quasars spanning 10 months to 3.7 years and found that $50 \%-60 \%$ BALs are variable on such timescales. Using multiepoch spectra from the SDSS I/II/III, Filiz Ak et al. (2012, 2013) made a statistical study of the largest sample consisting of 582 BAL quasars. They showed that the fraction of variable BALs increases with increasing observing intervals. All these studies also found that different BAL components of $\mathrm{C}_{\mathrm{IV}}$ in one object or the same troughs of $\mathrm{C}_{\mathrm{IV}}$ and $\mathrm{Si}$ IV BALs vary in a coordinated manner (strengthening or weakening). These coordinated variations can most simply be interpreted as caused by the variation in the ionizing continuum (Hamann et al. 2011). In contrast, Wildy et al. (2014) claimed that BAL variations were not correlated with continuum variations, casting doubt on this interpretation. In addition, Capellupo et al. (2014) found variable $\mathrm{P} v$ absorption lines associated with non-black $\mathrm{C}$ IV absorption lines, which they interpreted as ionized gas moving across the continuum source. There is also an argument that the ionizing continuum may vary in a different way from the observed UV continuum due to changes in a presumed high ionization shielding gas (Filiz Ak et al. 2012). That intervening gas filters hard ionizing photons to prevent overionized outer layers of gas (Murray et al. 1995). Also, in some cases, a new component appears or a previously observed trough disappears, which is interpreted as gas moving into or out of our line of sight (Hamann et al. 2008; Leighly et al. 2009; Krongold et al. 2010; Filiz Ak et al. 2012).

While the cause of this variability is still a matter of debate, there is no doubt that absorption-line variability can put useful constraints on the physical state and/or kinematics of the absorbing gas. In the case of photoionization, the variability timescale will set an upper limit on the ionization or recombination timescale, which depends solely on the flux density of the incident ionizing continuum or on the gas density. In the case of gas moving in and out of the line of sight, important constraints on the gas transverse velocity and the size of the clump can be set. In combination with specific kinematic models, this can translate into constraints on the distance of the absorber to the central black holes (e.g., Hall et al. 2011).

In this paper, we will examine the variability of BALs and mini-BALs and their correlations with continuum and emission-line variability to constrain the physical process that leads to such variability and to interpret the observed variability in the context of such a physical process. The paper is organized as follows. We describe the sample and data analysis methods in Sections 2 and 3. A correlation analysis is presented in Section 4. We discuss the implications and photoionization models in Section 5. A summary is given in Section 5.

\section{VARIABLE ABSORPTION LINE QUASAR SAMPLE}

We searched the SDSS DR10 archive for quasars that were observed two or more times. We merged the quasar catalog of SDSS data release 7 (DR7; Schneider et al. 2010) with that of DR10 (Pâris et al. 2014). Duplicated entries are removed. We compared this catalog with the SDSS spectroscopic catalog and selected quasars with multi-spectroscopic observations. To investigate the variability of $\mathrm{C}$ IV, Si IV, and $\mathrm{Nv}$, we adopt a redshift cut of $2.2<z \leqslant 4.7$. To ensure detection of major absorption lines, we only keep quasars with at least one spectrum with signal-to-noise ratio of $\mathrm{S} / \mathrm{N}>10$. After these screenings, we obtain a sample of 6250 quasars with two

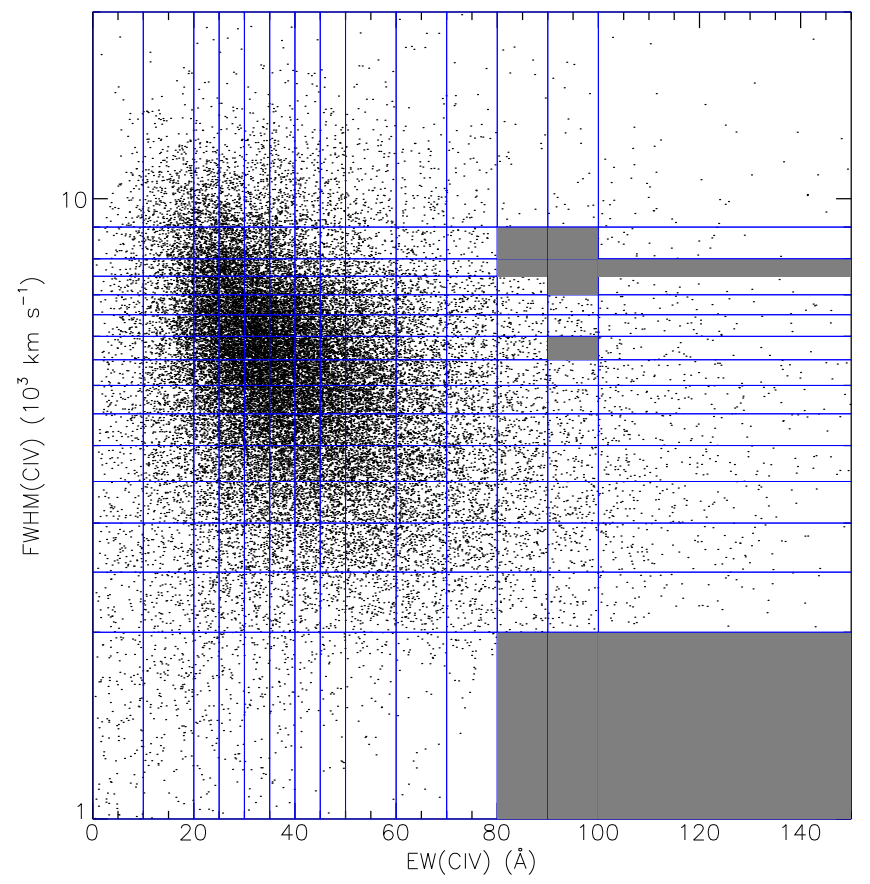

Figure 1. Grids on the plane of EW(C IV) vs. FWHM(C IV) of which unabsorbed composite spectra are made. The scattered dots represent the data of quasars in SDSS DR7. No composite spectrum is made for gray area because there are fewer than 20 quasars in each grid.

spectra or more. In the following subsection, we describe the method of construction of a variable absorption-line quasar sample.

\subsection{Unabsorbed Quasar Templates and Identification of Absorption Lines}

In order to identify absorption lines, we need a set of unabsorbed template quasar spectra. In the literature, there are several ways of constructing such templates: using quasar composite spectrum as an approximate (e.g., Weymann et al. 1991), using the reddened power-law continuum plus Gaussian or Lorentzian emission-line models (e.g., Filiz Ak et al. 2012), using a set of PCA spectra of quasars (e.g., Wildy et al. 2014), and finally adopting the best-matching unabsorbed quasars (Zhang et al. 2014; Liu et al. 2015). In this paper, we will use a set of composite spectra with different properties of emission lines to account for the diversity of quasar spectra. To first order, a quasar emission line can be described by its relative strength (EW), its blueshift, and its width (FWHM). Previous studies have shown that those line parameters for C IV are correlated with each other (e.g., Corbin \& Boroson 1996; Richards et al. 2011; Wang et al. 2011) and also with the relative strength of other lines (Wang et al. 2012). Here, we divide 38,377 non-BAL quasars by $1.5<z \leqslant 4.0$ and $\mathrm{S} / \mathrm{N}_{1350}>10$ in Data Release 7 (DR7) into different bins according to their line width (FWHM) and equivalent width (EW) of C IV as measured by Shen et al. (2011). We then make a composite spectrum for each bin. We split the EW(C IV) and FWHM plane into 210 unequal grids as shown in Figure 1. To ensure a sufficient number $(>20)$ of spectra per bin, we use a larger grid in the low-density zone of parameter space. Among the 210 grids, 10 contain less than 20 spectra each, so no composite spectrum is made for these grids. In the process, we mask bad pixels using the SDSS mask array and also mask 


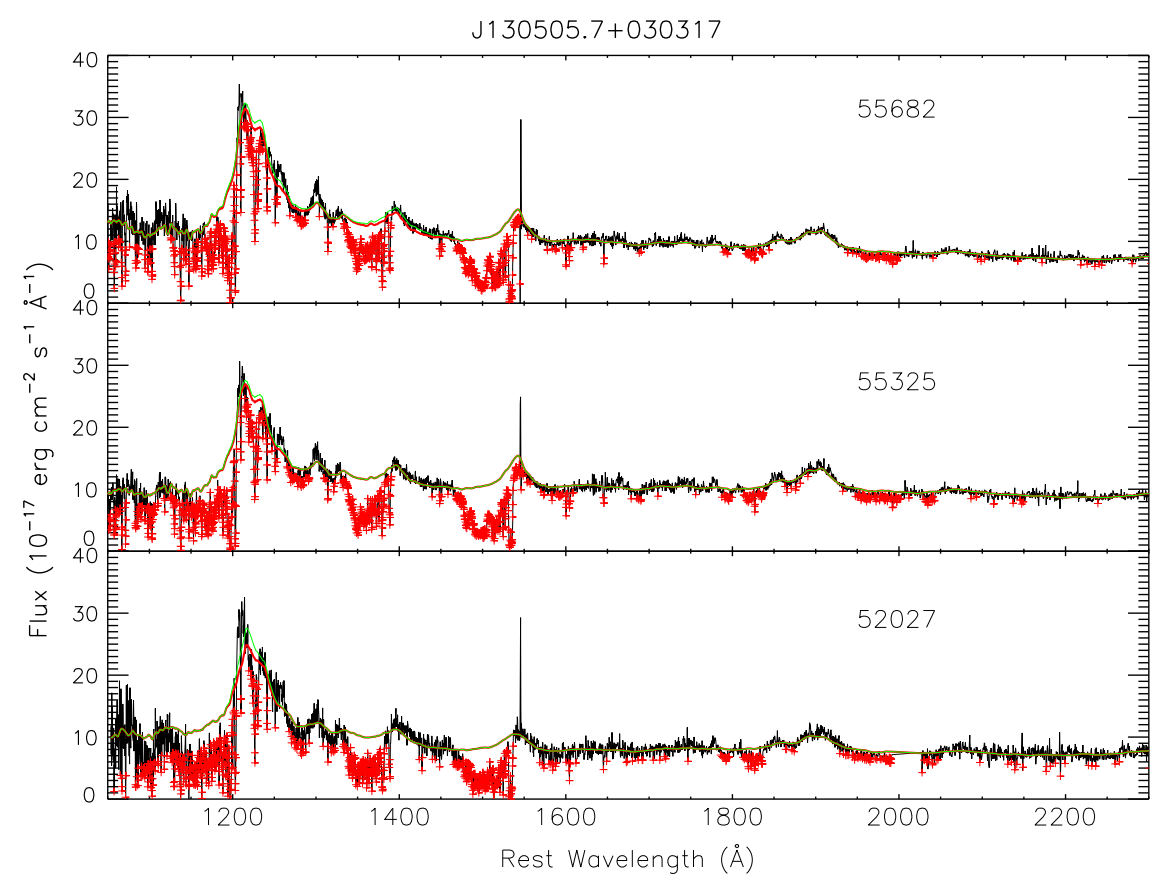

Figure 2. Unabsorbed template fit to the spectra of SDSS J130505.7+030317 at three epochs. SDSS $m j d$ of the spectrum is labeled in each panel. The red line shows the scaled best matched template, while the green line has additional Gaussians to account for emission lines. The red crosses are those pixels $3 \sigma$ below the fit, and thus are masked out during the fit.

strong narrow absorption lines in individual spectra. We smooth the SDSS spectrum using a Savitzky-Golay filter with 33 pixels, and a fourth-degree polynomial. Any region $10 \%$ lower than the smoothed spectrum and with a width of at least 3 pixels is considered a narrow absorption line. We replace the flux of these pixels with the smoothed ones. Finally, we check the templates by eye. This yields a total of 200 templates. With this number of templates, we are able to fit emission lines and continuum in individual objects.

We fit these templates to the spectra using a double powerlaw function as a scale factor,

$$
S_{\lambda}=A[1]\left(\frac{\lambda}{2000 \AA}\right)^{A[2]}+A[3]\left(\frac{\lambda}{2000 \AA}\right)^{A[4]} \text {, }
$$

where coefficients $A[1: 4]$ were determined by minimizing $\chi^{2}$. Note that the power law can also be used to describe the SMClike dust extinction in the UV band, which can be considered a good approximation for quasar reddening (e.g., Richards et al. 2003). The wavelength range of this fit covers from 1260 to $2750 \AA{ }^{5}$ We also shift the templates in wavelength up to 6 pixels to account for uncertainties in the redshift. To do the fit, we iteratively mask pixels significantly $(3 \sigma)$ lower than the model on the blue side of C IV, Si IV in order to exclude possible absorption lines. We select the 10 best templates according to the criterion of the maximum number of pixels within $1 \sigma$ error, penalized for the number of pixels exceeding the model at the $3 \sigma$ level. This usually yields a reasonable fit to the SDSS spectrum except, in some cases, around strong emission lines. To evaluate the fit around strong emission lines, we calculate the significance of excess or deficient flux around each line

\footnotetext{
5 Despite the relatively large number of templates used, in a significan number of cases, we cannot find a proper match for all strong emission lines from 1200 to $2850 \AA$.
}

$\left(\chi=\sum\left(f-f_{\text {fit }}\right) / \sqrt{\left(\sum \sigma_{f}^{2}\right)}\right)$, which is used as a criterion for the addition of a Gaussian for the excess or deficient emission line. In the case of the deficient regions, $\chi$ is only calculated on the red side of an emission line for $\mathrm{C}$ IV, $\mathrm{N} \mathrm{v}$, and $\mathrm{Si}$ IV because redshifted absorption lines are very rare (Hall et al. 2013). We then extend it blueward. If it is significant at the $3 \sigma$ level, a Gaussian function is then added to the selected best fit, and a new fit is performed. About $8 \%$ of spectra need an additional Gaussian component for $\mathrm{C}$ IV emission line. In most cases, this can reproduce a reasonably good fit as judged by eye. Examples of fits are shown in Figure 2. For convenience, we will term this best fit "matching template," although it may include additional Gaussians. A normalized spectrum is created by dividing the observed spectrum by the matching template.

We searched contiguous deficient pixels for intrinsic absorption lines in the normalized spectrum. With a focus on the moderate to broad absorption lines, which are more likely intrinsic, deficiency over a width of $\Delta \ln \lambda \geqslant 10^{-3}$, or $300 \mathrm{~km} \mathrm{~s}^{-1}$ in velocity and more statistically significant than $5 \sigma$, is screened. The significance is defined by the total deficient flux divided by the square root of the summed errors. Then, we check by eye to exclude false ones caused by an improper fit in most cases. In a number of cases, $\mathrm{C}$ IV absorption lines move to wavelengths shorter than $1400 \AA$, so these may be identified incorrectly as Si IV absorption lines. To deal with this, we simultaneously check the absorption lines in C IV, Si IV, and N v over a velocity range up to $40,000 \mathrm{~km} \mathrm{~s}^{-1}$, if $\mathrm{Nv}$ is within the spectral coverage. ${ }^{6}$ Our assumption is that any $\mathrm{Si}$ IV absorption lines must have an accompanying $\mathrm{C}$ IV. If not, the feature will be identified tentatively as a high-velocity

\footnotetext{
6 In about $10 \%$ of cases, $\mathrm{N} \mathrm{v}$ is shifted out of the SDSS spectral coverage because the blueshift of the absorption line is too large or because the $\mathrm{N} v$ absorption line falls in a problematic spectral range as flagged by the SDSS mask array by chance.
} 


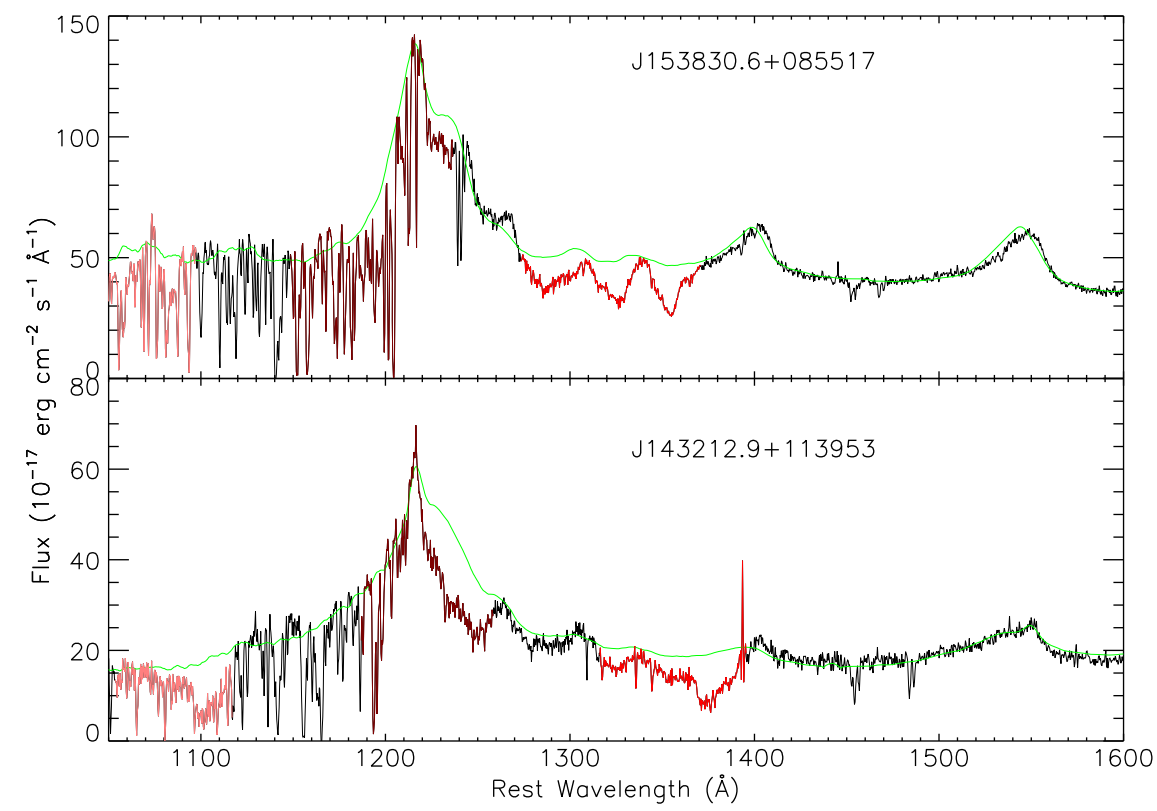

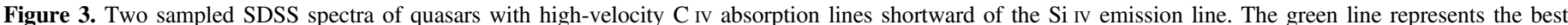

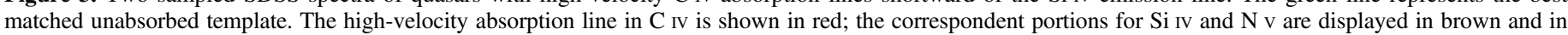
pink, respectively.

C IV component. For confirmation, we look for a possible corresponding $\mathrm{N} v$ and $\mathrm{Si}$ IV absorption line. Examples of highvelocity absorption lines are shown in Figure 3. Note that we do not intend to select a complete sample of BAL or mini-BAL quasars, but rather establish a starting point for finding a reliable sample of variable absorption-line quasars.

\subsection{Identification of Variable Absorption Lines}

In order to determine whether emission or absorption lines are variable or not, with respect to the continuum, we first select the highest $\mathrm{S} / \mathrm{N}$ spectrum of the quasar as a reference and then rescale it using the double power-law function (Equation (1)), as in the template fit, to match the quasar spectrum in the overlapping region obtained at other epochs. This is an empirical approach to take into account for potential variations of the continuum shape in which the spectrum usually becomes bluer as continuum brightens and for the uncertainty in the relative spectrophotometric calibration (Dawson et al. 2013 and the Appendix). As shown below, with this recipe we can fit the observations very well. Examples of the fit are displayed in the Figure 4.

Once this rescaling is done, we found that weak lines and the continuum spectral energy distribution (SED) match very well in the two epochs. The median scatter in the difference spectrum is comparable to the combined uncertainties (square root of the sum the square of the flux errors) of the spectra provided by SDSS in the regions avoiding strong emission lines. However, emission lines and absorption lines show significant differences in many cases. To account for variations of emission-line EWs, we then add/subtract a Gaussian to/ from the rescaled spectrum. To minimize potential spurious results, the sign of normalization, which will be used as an indicator of the increase or decrease of overall emission-line EW in Section 3, is assumed to be the same for all emission lines. We also restrict the center of the Gaussian to lie within $\pm 500 \mathrm{~km} \mathrm{~s}^{-1}$ of the line center at the source rest frame. Although the actual variation of emission lines may be more

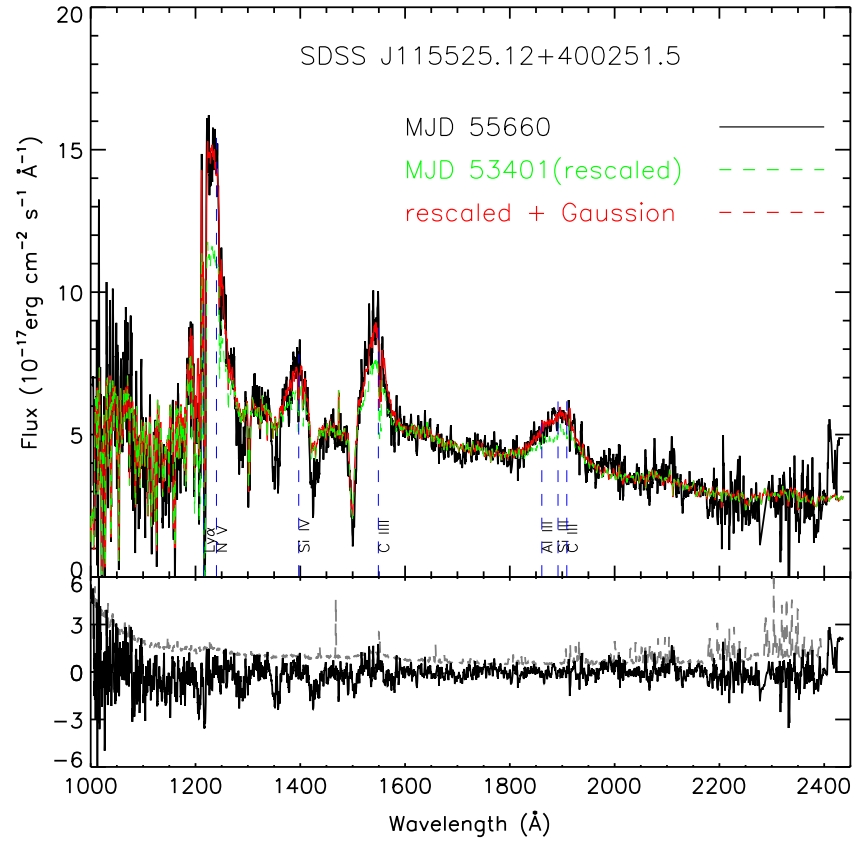

Figure 4. Match one SDSS spectrum (in black) to the reference spectrum taken at another epoch by multiplying one spectrum with the double power law described in the text (the green line). The red curve represents the one with additional Gaussians to account for the change of the emission-line equivalent width. The residuals of fits (solid line) and the combined spectrum uncertainties (dashed line) are plotted in the lower panel.

complicated, for the $\mathrm{S} / \mathrm{N}$ of our spectra, this reproduces good fits for almost all lines. With this fit, we found that the scatter in the difference spectrum over emission-line regions is similar to that of continuum regions. In comparison with unabsorbed quasar template matching, the rescaled reference matching usually produces a better fit outside the absorption-line region. Therefore, in the following, we will measure the absorption- 


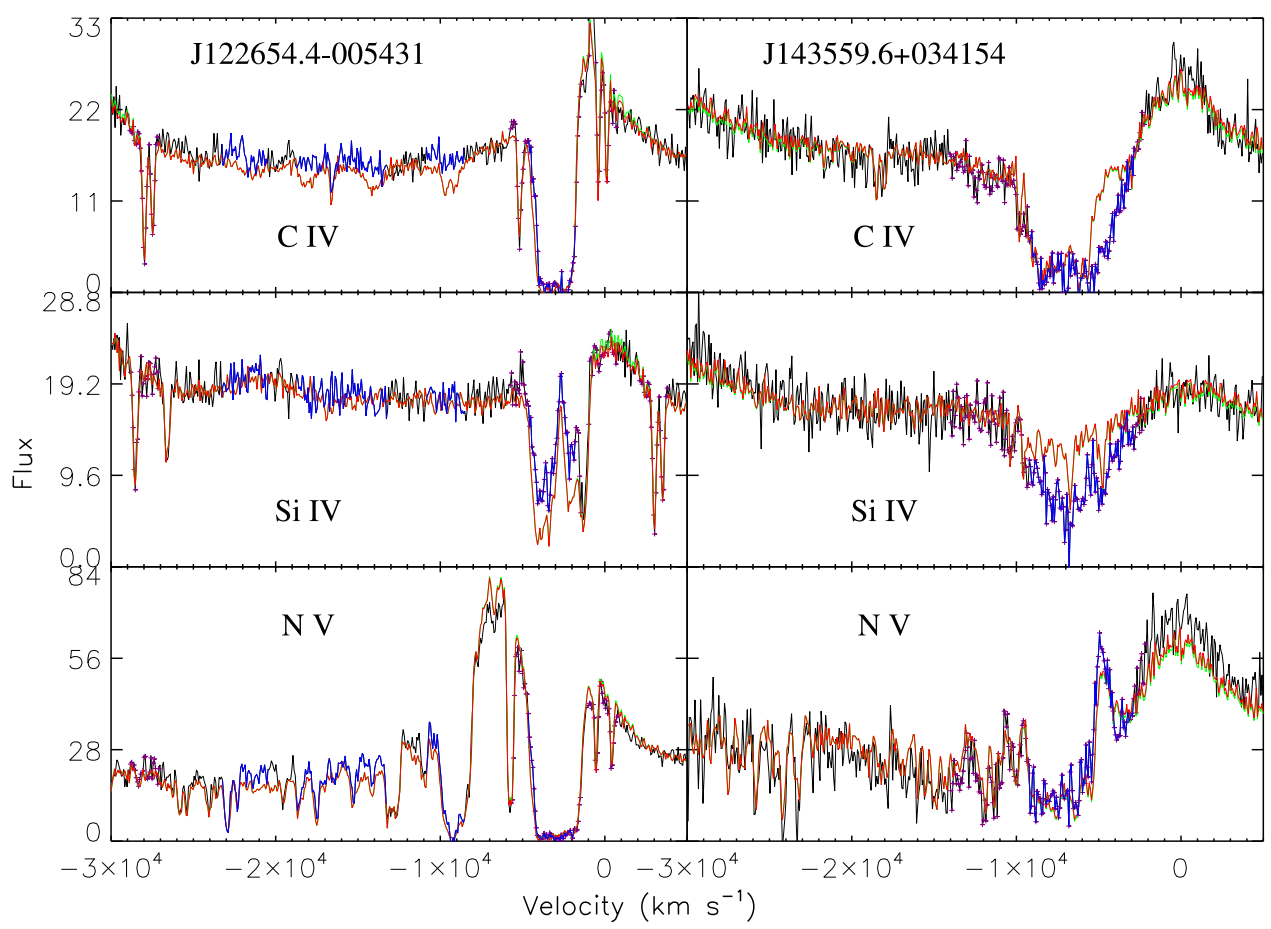

Figure 5. Different variations of $\mathrm{C}$ Iv, $\mathrm{Si}$ Iv, and $\mathrm{N} v$ absorption lines in two quasars. The variable regions in one or more absorption lines are marked in blue. Other colors are the same as Figure 4. Noting strong $\mathrm{C}$ IV and N v, absorption lines are not apparently variable in the deep trough while the weaker absorption-line Si Iv is. This is likely an observation effect with which it is more difficult to detect small flux changes in the deep absorption trough caused by ionic column density variations. The absorption trough around $-9000 \mathrm{~km} \mathrm{~s}^{-1}$ in the lower left panel is due to Ly $\alpha$ absorption.

line variability from the difference spectrum rather than by comparing EWs obtained in the template fitting.

To identify variable absorption-line components in the difference spectrum, we searched for contiguous negative and positive bins to determine the range of variable components. The uncertainties are estimated by taking into account the flux uncertainties of the two spectra given by the SDSS pipeline and possible systematic uncertainties due to rescaling. The latter is estimated in neighboring regions that are outside of the absorption troughs. Because difference spectra do not have a high $\mathrm{S} / \mathrm{N}$, in general, a single variable component may split into two or more segments due to statistical fluctuations in the spectrum. To overcome this problem, we take three steps. First, we mark all pixels where the difference is larger than $5 \%$ of the average value and more than $3 \sigma$. Adjacent marked pixels are then connected to form a variable region. Next, we expand such a regime into neighboring pixels that have the same sign in the difference spectrum but are at the less than $3 \sigma$ significant level. After that, we also merge the neighboring regions with the same variable sign and with a separation of less than four pixels. Finally, we identify the same component in different epochs, and then we extend the velocity range of each component to cover the component at all epochs.

In practice, we iterate three times through the above procedures. We mask the variable absorption regions identified in the previous iteration, rescale the spectrum, and refit the emission lines. After that, we redefine the variable absorptionline region. Finally, the sample is then examined by eye and spurious regions are excluded. In the end, a sample of 452 quasars with detected variable absorption lines were identified. We list the sources and regimes of the variable absorption lines in Table 2. The maximum velocities of the $\mathrm{C}$ IV absorption-line troughs in this sample extend to up to $45000 \mathrm{~km} \mathrm{~s}^{-1}$, and the widths of individual absorption troughs are in the range of $500-15000 \mathrm{~km} \mathrm{~s}^{-1}$. Note that variable components may be the entire trough or just a portion of the trough. The latter does not necessarily mean that the variable portion of the trough is physically different from the rest, instead it may reflect the limitation that we cannot detect small variations caused by even moderate changes in the ion column density in certain circumstances. This is evident especially in the deep trough of C IV, where we sometimes observe an apparent variation of the Si IV absorption line while no similar $\mathrm{C}$ IV variation is seen (Figure 5; and also Filiz Ak et al. 2013). In comparison with those of Filiz Ak et al. (2013), our sample also includes absorption lines that do not meet the criteria of BALs. The basic properties of the sample are summarized in the Table 1.

\section{MEASURING THE VARIABILITY PARAMETERS AND STATISTICAL METHOD}

In this paper, we will use the sign of the variation with respect to the scaled reference spectrum as a non-parametric description of absorption-line variability. We simply measure the integrated variable flux over the variable region of absorption lines in the difference spectrum using the reference-matching method. We assign a sign of absorption-line variation of +1 when the absorption-line trough becomes significantly deeper (at the $3 \sigma$ level), -1 when the trough gets significantly shallower, and 0 otherwise. In comparison with previous measurements using variations of an absorption-line $\mathrm{EW}$, our method avoids the additional uncertainty introduced in the process of template matching while measuring the EWs of absorption lines in each spectrum.

We estimate the variability of the continuum and emission lines in order to explore the internal driver for absorption-line variability. It is known that the spectrophotometric calibration 
Table 1

Basic Statistics of the C IV Variable BAL Sample

\begin{tabular}{lc}
\hline \hline Variable Parameter & Number of Sources \\
\hline C IV & 452 \\
Si IV & 194 \\
N v & 227 \\
Emission Lines & 396 \\
Continuum & 421 \\
\hline
\end{tabular}

of SDSS has an uncertainty of about $\sigma_{r}=0.05 \mathrm{mag}$ in the SDSS I/II surveys (Abazajian et al. 2009) and is considerably worse for BOSS spectra (Dawson et al. 2013; Pâris et al. 2014). However, as shown in the Appendix, the distribution of difference magnitudes between two epochs of our detected variable absorption-line quasars is considerably broader than possible calibration uncertainties. Hence, it is still possible to extract statistically useful information about the sign of continuum variability. We consider a continuum variation significant when its amplitude is greater than $5 \%$ at the $g$-band or, typically, $\sim 1400 \mathrm{~A}$ in the quasar rest frame. As for absorption lines, we assign a sign of the continuum variation to +1 when continuum flux around $1400 \AA$ is more than $5 \%$ brighter than the reference spectrum, -1 for the opposite case, and 0 otherwise.

If there are only two categories, the concordance index should follow a binomial distribution with a probability of $P(x>k ; n, p)=\Sigma_{i \leqslant k} C_{n}^{i} p^{i}(1-p)^{(n-i)}$, for a result of more than $k$ concordant cases in $n$ pairs with a concordant probability of $p$ and anticoncordant probability of $1-p$ for each pair, where $C_{n}^{i}$ is the binomial coefficient. In our case, the concordance index can take an additional value of 0 because of an undefined variability sign of one variable, which does not affect our analysis. Because undefined variability signs can be caused by a low signal-to-noise ratio of the spectrum or a small variability, which are not of interest to us, we seek the probability $P(x>k ; n, p)$ among $n$ of those pairs with defined variability signs of both variables. This probability follows a binomial distribution. If we take $p=0.5$, then $P$ gives the chance coincidence that concordance and anti-concordance have equal possibilities. Furthermore, one can derive the most likely value of $p=k / n$ and $1 \sigma$ error in $p$ as $\sqrt{p(p-1) / n}$ if there is no measurement error in the concordance index. On the other hand, if the concordance index were misassigned randomly for a fraction $q$ of sources, we can derive easily $p=(k / n-q) /(1-2 q)$ by considering those misassigned events. The error can be estimated by Monte Carlo simulations. It is evident that taking into account the misassignments will make $p$ larger if $p>0.5$ and smaller if $p<0.5$. This is reasonable because random errors would only smear the difference rather than enhance it, making apparent $p$ closer to 0.5 .

In light of the well-established intrinsic Baldwin effect, i.e., the EW of an emission line correlates negatively with that of the continuum during continuum variations (e.g., Kinney et al. 1990), we will use the variation of emission-line EWs as an independent check for the sign of continuum variation. Lags in the emission line to continuum variations will introduce a correlation similar to the Baldwin effect. We calculate variations of the EWs of strong emission lines (including

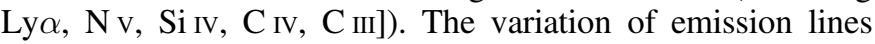
relative to the continuum is measured in the reference-matched spectrum, and the sign of emission-line variations can be directly extracted from the sign of additional Gaussian components in the reference-matched spectrum. Similarly, we assign a sign for the emission-line variation: +1 if the line flux in the reference-matching spectrum increased significantly and -1 if the line flux decreased significantly, and 0 otherwise.

Among 510 spectrum pairs of 452 objects (Table 1), 292 pairs show opposite signs of continuum and emission-line EW variability, 111 pairs display the same sign, and the rest of the 107 pairs do not have a well-defined variability sign of either continuum or emission lines. Therefore, in a total of 403 cases with well-defined variability sign in both continuum and emission lines, 292 have a concordance index of -1 . Following the binomial distribution, we obtain a probability concordance index of $-1: p=0.725 \pm 0.022$, which is $10.2 \sigma$ from no preference $(p=0.5)$ for the anticorrelation between continuum and emission-line variability. Therefore, the intrinsic Baldwin effect is detected at a high confidence level for the highluminosity quasars.

\section{CORRELATED VARIATIONS OF ABSORPTION LINES, EMISSION LINES, AND CONTINUUM}

\subsection{Correlated Variations of Absorption Lines}

Previous observations have shown that different components of the $\mathrm{C}$ IV absorption line show coordinated variations, i.e., in the same sign of variations (see references in Section 1). This is confirmed by our study. We define a concordance index with +1 for the all components that vary with the same sign and -1 for one or more components with opposite signs. Among 114 spectral pairs of 101 objects with multiple variable $C_{\text {IV }}$ components, only 25 pairs display one or more components varying in opposite sign to others, while the rest show the same sign. This gives a probability for concordant variations of $p=0.781 \pm 0.039$, which is $7.2 \sigma$ from $p=0.5$ following the binomial distribution. Note that when only the most significant variation is selected for each object, if there is more than one spectral pair, 97 of 101 objects show concordant variations of different components, consistent with the above conclusion. Furthermore, we notice that in the case of incongruent variations, the two asynchronous components often share an absorption trough, indicating an acceleration or deceleration of an absorption-line component. This will be studied in detail in a forthcoming paper.

Next, we explore the coherence of the variations of the same component of different absorption lines. It was demonstrated by several previous authors that variations of $\mathrm{C}$ IV and $\mathrm{Si}$ IV absorption lines are highly concordant (see references in Section 1). Figure 6 shows the distributions of the concordance index for ( $\mathrm{C}$ IV, $\mathrm{Si}$ IV) and ( $\mathrm{C}$ IV, $\mathrm{N}$ v) pairs. In cases of more than one variable $\mathrm{C}$ IV absorption-line component, for each line we sum up the sign of individual components and assign a sign of +1 for a positive sum, -1 for a negative sum, and 0 otherwise. Using this sign, we calculate the concordance index for the spectrum. We do count different spectra of one quasar as independent. However, absorption lines that are affected by bad pixels or are out of the spectral coverage were not counted. From these distributions, there are only a small fraction $(<4 \%)$ of cases where the two lines vary in an opposite way, although there is still a large fraction where $\mathrm{Si}$ IV or $\mathrm{N} \mathrm{v}$ does not vary significantly. $\mathrm{C}$ IV and $\mathrm{Nv}$ are correlated better than $\mathrm{C}_{\mathrm{IV}}$ and $\mathrm{Si}$ IV, i.e., $28 \%$ of pairs have a concordance index of 0 for $\mathrm{C}_{\mathrm{IV}}$ 

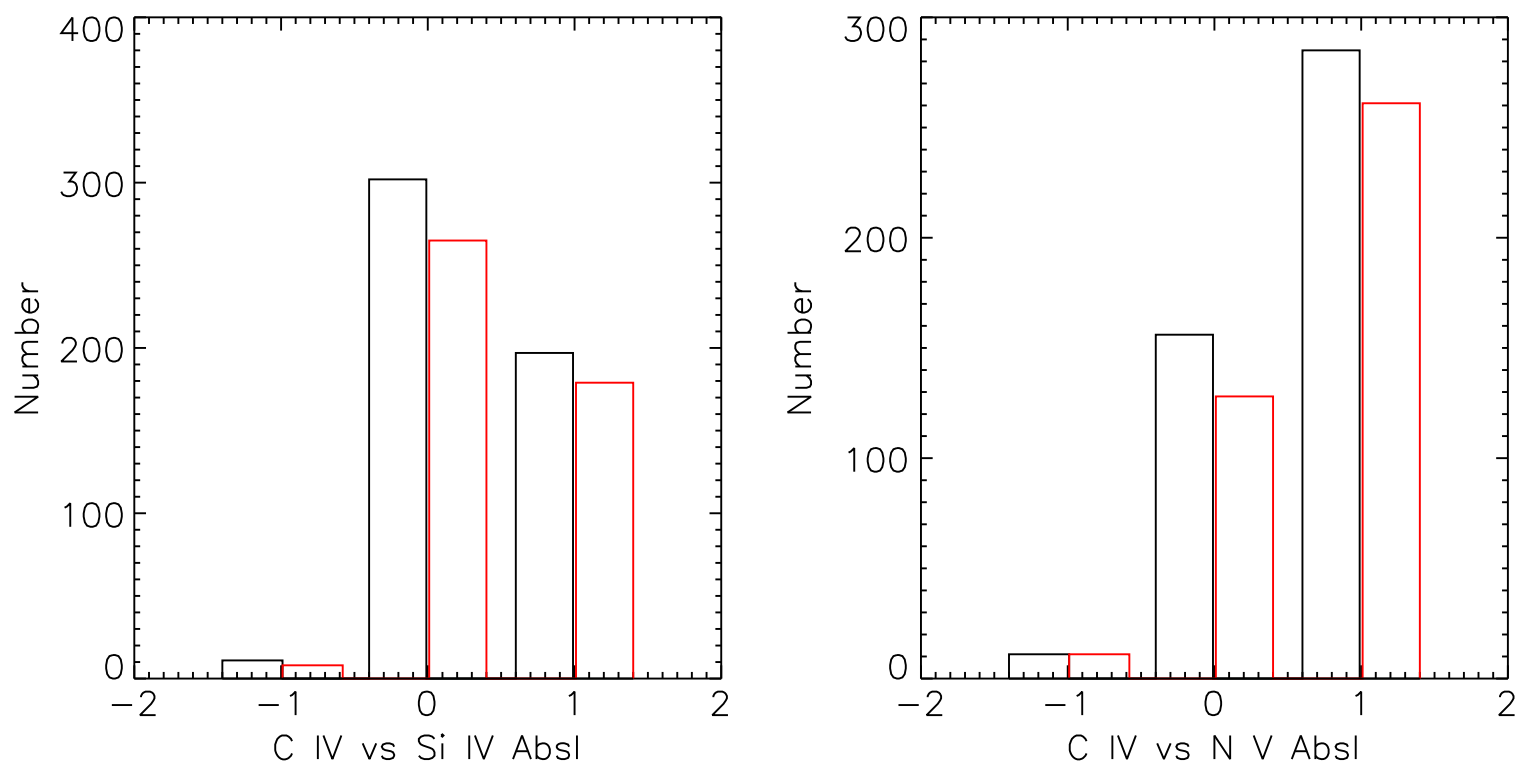

Figure 6. Distribution of the concordance index for the same variable component of different lines (left panel: $\mathrm{C}$ iv vs. Si Iv, right panel: $\mathrm{C}$ IV vs. $\mathrm{N}$ v). The concordance index is defined as +1 if both lines vary in the same sign (increasing or decreasing), -1 in opposite sign, and 0 otherwise. We exclude 58 spectra pairs and 52 quasars from analysis that $\mathrm{N} v$ absorption lines are outside of spectral coverage. The black boxes are the distribution of all spectra pairs in Table 1 , while the red boxes are based on the most significant pair for each object.

versus $\mathrm{Nv}$, while $60 \%$ are between $\mathrm{C}$ Iv and Si Iv. Using the distributions in Figure 6, we calculate $p=0.944 \pm 0.015$ (black boxes; $0.949 \pm 0.017$ for red boxes) for concordant variations of $\mathrm{C}$ IV and $\mathrm{Si}$ IV absorption lines. The probability for concordant variation of $\mathrm{C}$ IV and $\mathrm{N} v$ is $p=0.963 \pm 0.010$ for the black boxes in the right panel of Figure 6 and $0.960 \pm 0.012$ for the red boxes.

A close examination of the cases of concordance index 0 shows that, in most cases, the non-variable line ( $\mathrm{N} v$ or $\mathrm{Si}$ Iv) either has a very shallow or very deep absorption trough. So the non-detection of variability is most likely due to the insensitivity of the variation of the flux in absorption trough to changes in the ion column density in these cases and the relatively low $\mathrm{S} / \mathrm{N}$ ratio of SDSS spectrum. This explains why more sources in the $\mathrm{Si}$ IV and $\mathrm{C}$ IV pairs have a concordance index of 0 than $\mathrm{C}$ Iv and $\mathrm{N} v$ pairs. The latter pairs have similar optical depths, while $\mathrm{Si}$ Iv usually has a considerably smaller optical depth. In fact, Si Iv variations are observed more frequently in quasars with a deep C IV absorption trough. We will explore the implication of this in the next section.

\subsection{Coordinated Variations between Absorption Lines and Continuum/Emission Lines}

Correlations between variations of absorption lines and continuum/emission lines provide important insight into the origin of absorption-line variability. However, previous studies have not reached a consensus on whether absorption-line and continuum variations are correlated or not. Gibson et al. (2008) found that the correlations between the variations of absorption-line EWs of $\mathrm{C}$ IV and continuum flux is not significant for 14 BAL quasars. In a recent study, Vivek et al. (2014) did not find any trend in the variation of the $\mathrm{Mg}$ II and $\mathrm{Al}$ III BAL EWs with the variations of continuum parameters. In a recent study, Wildy et al. (2014) argued that variations of $\mathrm{Si}_{\text {IV }}$ and C IV BALs are not driven by photoionization based on the lack of correlations with continuum luminosity. We only consider the signs of absorption, emission line, and continuum variations in this subsection because the exact amplitude of continuum variability is rather difficult to quantify with only SDSS spectra in view of uncertainties in the absolute flux calibration. Since $\mathrm{C}_{\mathrm{IV}}$, Si Iv, and Nv absorption lines show the same sign of variations, for each absorption component, we count only the sign of significant variation in the $\mathrm{C}$ IV line and compare this with continuum variations. As in the last subsection, we assign a concordance index of +1 for the case where an absorption line becomes stronger when continuum brightens and -1 in the opposite way, while 0 for the case in which the sign of continuum variation is not determined. We also define a similar concordance index to quantify the coherence between the variations of absorption lines and emission-line EWs. We show our results in Figure 7.

Obviously, the variations of absorption lines are statistically highly coordinated with those of the continuum and EWs of emission lines. The apparent probability for concordant variations of emission and absorption line is $p=0.796 \pm 0.017$, which is $17.4 \sigma$ for $p=0.5$. We get the same result if only the most significant variation for each source is considered (using the red histogram of Figure 7). According to the intrinsic Baldwin effect, the two results suggest that absorption lines weaken as the continuum brightens and vise versa. Similarly, the apparent probability for concordant variations of continuum and absorption lines is $p=0.726 \pm 0.016$, which is $14.1 \sigma$ from $p=0.5$. We obtain a similar result, $p=0.726 \pm 0.022(10.3 \sigma)$, when only the most significant variations are considered for each source. Considering possible misassigned signs for the variations of either variables, the real $p$ should be higher.

The conclusion on the correlated variations of absorption line and continuum should be not affected by the SDSS flux calibration uncertainty of SDSS spectra. Because the spectrophotometric calibration error is not expected to correlate with the variation of the absorption-line strength, any calibration errors will only diminish rather than enhance the correlated variations. Thus, the observed coordinated variations of 

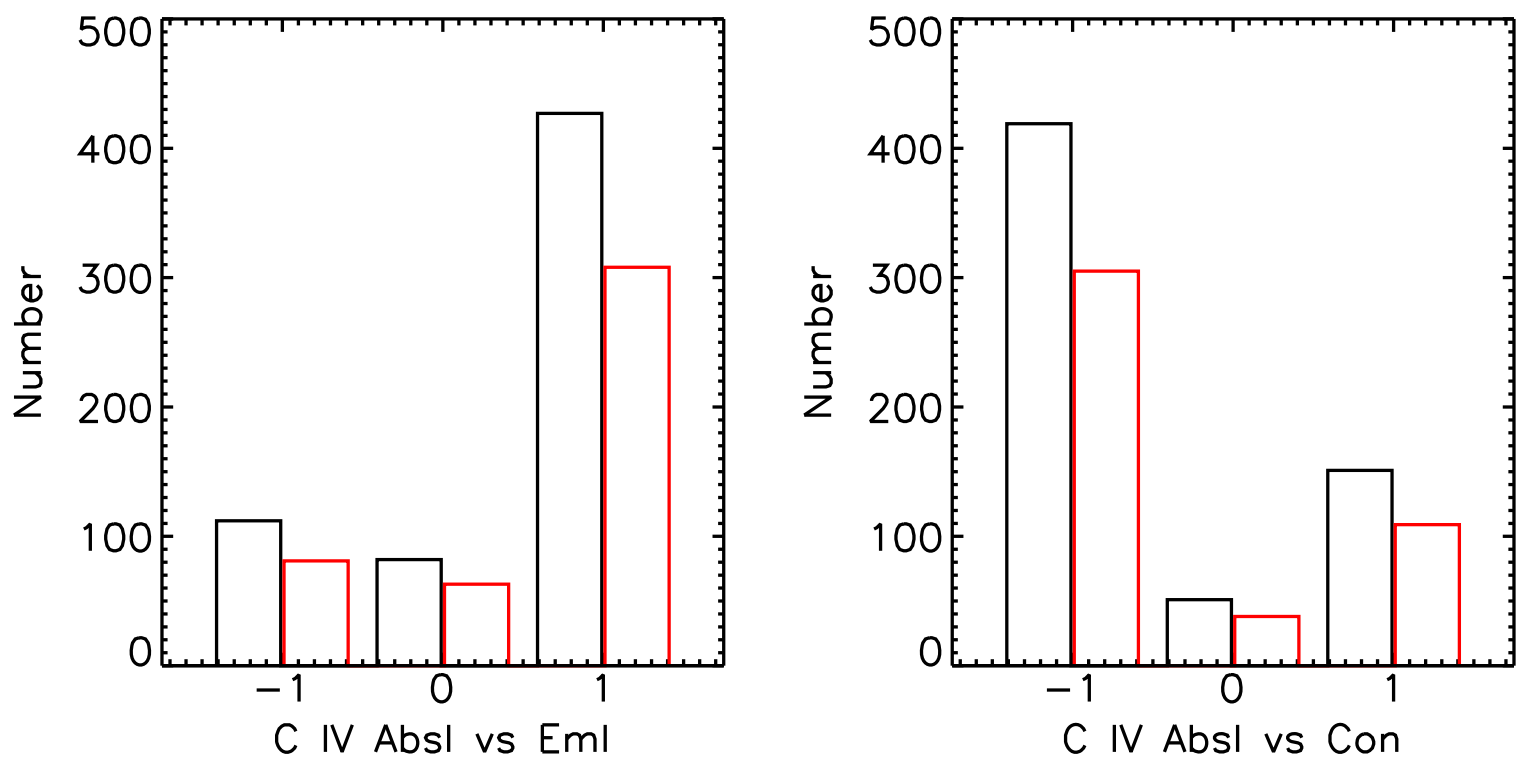

Figure 7. Distribution of the concordance index for $\mathrm{C}$ IV absorption line vs. continuum (right panel) and $\mathrm{C}$ iv absorption line vs. emission line (left panel). The concordance index is defined as +1 if the absorption and emission lines weaken or strengthen simultaneously, -1 if one weakens and the other strengthens, and 0 otherwise. Similarly, in the case of continuum vs. absorption line, a concordance index of +1 means the absorption line strengthens while the continuum brightens and vice versa. Only quasars with variable $\mathrm{C}_{\mathrm{IV}}$ absorption lines are included. See the text for a definition of this index. Colors are as in Figure 6.

absorption lines and continuum must be real. This is further supported by coordinated variations between absorption-line and emission-line EW, which are not affected by the flux calibration. Note that we use the continuum flux variability amplitude of $>5 \%$ as a threshold, while it is estimated conservatively in the Appendix that about 13\% have been assigned to an opposite sign of continuum variations and an additional $10 \%$ with an undefined sign of variations. The total number is comparable to the total fraction (33\%) of sources with a concordance index of 0 or 1 . Thus, our result is consistent with the idea that a large fraction of cases with a concordance index of 0 or -1 are due to absolute flux calibration uncertainties. If we take the numbers given above, about $14.4 \%$ of BAL quasars were assigned to a wrong sign of continuum variation among those with defined signs. According to the equation in Section 3, we obtain $p=0.817$. However, the exact probability has to wait until a complete solution of the flux calibration uncertainties are found. We see that there are fewer cases of concordance index -1 in the absorption- and emission-line pairs because there is no such calibration problem, although the measurement of the variation of emission-line EW is subject to larger statistical uncertainties.

There are rare cases where a new component of an absorption line emerges or a previously known component disappears (Hamann et al. 2008; Leighly et al. 2009; Krongold et al. 2010; Filiz Ak et al. 2012). They are extreme cases where the absorption-line trough is below the detection limit in one epoch, which depends on the $\mathrm{S} / \mathrm{N}$ of the spectrum and the correctness of the template used to match the spectrum. These cases are often taken as evidence for outflows moving in and out of the line of sight. The large sample of multi-epoch spectroscopic quasars enables us to extract a statistically significant sample of about 70 spectral pairs of around 50 quasars with emergence or disappearance of at least one absorption-line component (see Table 2 and Figure 8). We performed the same analysis of the concordance index among variations of continuum and absorption lines, or emission and absorption lines. The results are shown in Figure 9. The coordinated variations of absorption lines, continuum, and emission lines are statistically significant at 5.5 (for all pairs, black boxes in the figure) or $5.8 \sigma$ (only the most significant pair for each object, red boxes) and 5.2 (all pairs) or $4.8 \sigma$ (only one pair for each object), respectively. It is apparent that these cases also follow the above correlations, suggesting that the same physical process also accounts for these extreme variations of ion column density. Because they account for only a small fraction of our variable absorption line quasar sample, they do not affect the statistical results of the parent sample presented above.

\section{DISCUSSION}

\subsection{A Short Summary of Previous BAL Variability Studies and Our Results}

Previous works found that variations of BALs on the timescales of years are common $(50 \%-60 \%)$ and that the variable fraction and variability amplitude increase with the time interval of observations (Gibson et al. 2008; Capellupo et al. 2012; Filiz Ak et al. 2013). These authors also found that variations usually take place only in a narrow and relatively shallow portion of a broad BAL trough, although it is not clear how much this is due to saturation of the absorption line (see also Lundgren et al. 2007). The distribution of EW variations can be described using a random walk model (Filiz Ak et al. 2013). It has also been established that variations of $\mathrm{C}$ IV and $\mathrm{Si}$ IV are highly coordinated as are variations of different BAL components of C Iv (Capellupo et al. 2012; Filiz Ak et al. 2012, 2013). Disappearances or emergences of BAL components are observed in a small fraction of BAL quasars on a timescale of years (Hamann et al. 2008; Leighly et al. 2009; Filiz Ak et al. 2012; Rodríguez Hidalgo et al. 2013), and Filiz Ak et al. (2013) suggested that these are the extreme cases of BAL variability rather than a new phenomenon based on the overall distribution of the variability fraction of the absorption-line EW.

In this paper, first, we extended the coordinated variations of different lines to $\mathrm{Nv}$ and $\mathrm{C}$ IV absorption lines. Second, we 
Table 2

List of Variable Absorption Line Components

\begin{tabular}{|c|c|c|c|c|c|c|c|c|c|}
\hline \multirow[t]{2}{*}{ Name $^{\mathrm{a}}$} & \multirow[t]{2}{*}{ Plate } & \multirow[t]{2}{*}{ mjd } & \multirow[t]{2}{*}{ fiberid } & \multirow{2}{*}{$\begin{array}{c}\text { Velocity Range } \\
\quad\left(\mathrm{km} \mathrm{s}^{-1}\right)\end{array}$} & \multicolumn{5}{|c|}{ Variability Sign Relative to Reference ${ }^{\mathrm{b}}$} \\
\hline & & & & & $\overline{\mathrm{C}_{\text {IV }}}$ & Si IV & $\mathrm{Nv}$ & Continuum & Emission Line \\
\hline $\mathrm{J} 000330.18+000813.2$ & 4217 & 55478 & 532 & reference & - & - & - & - & - \\
\hline \multirow[t]{3}{*}{ J000951.17+092710.5 } & 4534 & 55863 & 968 & reference & - & - & - & - & - \\
\hline & 5648 & 55923 & 386 & $-25700 \sim-19200$ & 1 & 0 & 0 & -1 & 1 \\
\hline & 5648 & 55923 & 386 & $-14500 \sim-11100$ & 1 & 0 & 1 & -1 & 1 \\
\hline \multirow[t]{3}{*}{$\mathrm{J} 001130.55+005550.7$} & 4217 & 55478 & 948 & reference & - & - & - & - & - \\
\hline & 686 & 52519 & 603 & $-4900 \sim-700$ & 1 & 0 & 0 & -1 & 1 \\
\hline & 687 & 52518 & 339 & $-4900 \sim-700$ & 1 & 1 & 0 & 0 & 1 \\
\hline \multirow[t]{2}{*}{ J001818.70+002709.1 } & 4218 & 55479 & 972 & reference & - & - & - & - & - \\
\hline & 1491 & 52996 & 589 & $-16500 \sim-7100$ & 1 & 0 & 1 & 1 & -1 \\
\hline \multirow[t]{2}{*}{ J002146.71-004847.9 } & 4219 & 55480 & 216 & reference & - & - & - & - & - \\
\hline & 390 & 51816 & 161 & $-22300 \sim-19900$ & 1 & 0 & 0 & -1 & 1 \\
\hline $\mathrm{J} 003135.57+003421.2^{*}$ & 3587 & 55182 & 570 & reference & - & - & - & - & - \\
\hline
\end{tabular}

Notes.

a Symbol “*” marks an object with an appearance or disappearance of one or more absorption-line components with respect to the reference spectrum.

b The value 1 represents the case where either the equivalent width of an absorption line or emission line strengthens or the continuum brightens in comparison with the reference spectrum, -1 where an absorption or emission line weakens or continuum dims, 0 for insignificant variation, and - for no available data.

(This table is available in its entirety in machine-readable form.)

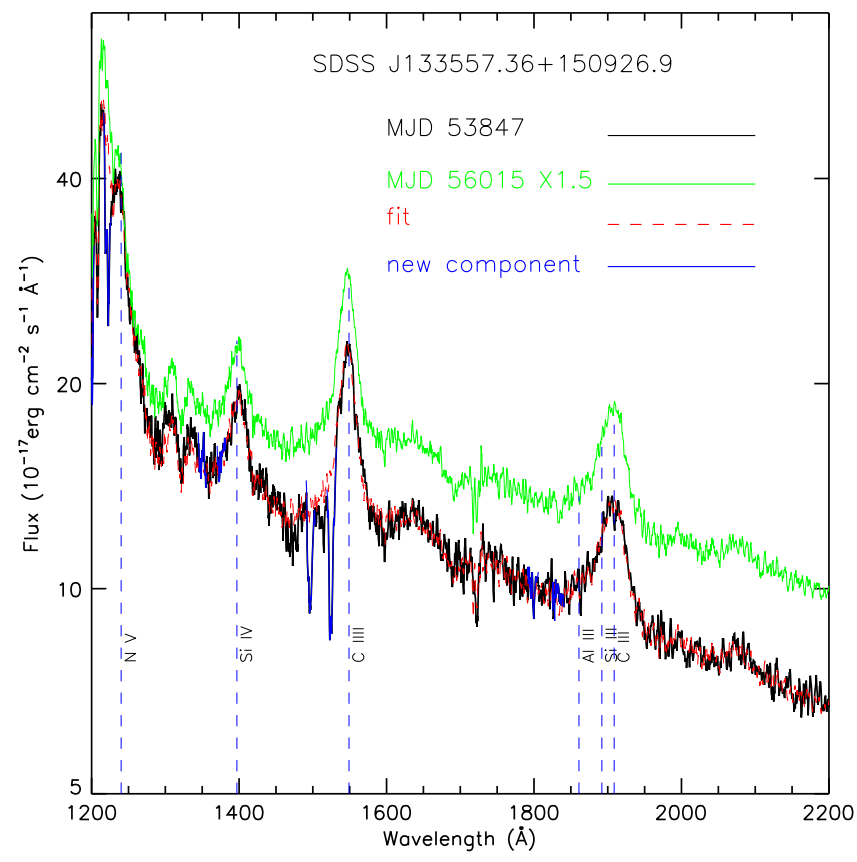

Figure 8. Example of emergence or disappearance of new BAL components. The SDSS spectra at two epochs are shown as black and green lines, while the best-fitted template is overplotted in red. Two new C Iv absorption-line components are marked in blue. The corresponding spectral region of Si IV, $\mathrm{N} \mathrm{v}$, and $\mathrm{Al} \mathrm{III} \mathrm{are} \mathrm{also} \mathrm{shown} \mathrm{in} \mathrm{blue.} \mathrm{The} \mathrm{new} \mathrm{absorption-line} \mathrm{components} \mathrm{are}$ also visible in $\mathrm{N} \mathrm{v}$.

showed that variations of absorption lines are highly coordinated with continuum variations. Finally, the emergence or disappearance of an absorption-line component is correlated with dimming or brightening of the UV continuum. Regarding the second point, it should be pointed out that data in previous studies are consistent with our results, although they were interpreted in a different ways. Barlow et al. (1992) found marginal evidence for a correlated continuum and absorptionline variations; Gibson et al. (2008) did not find significant correlations between the variations of the EWs of C Iv BALs with those of continuum for a sample of 13 BAL quasars observed in LBQS and SDSS. However, their result does not contradict ours. Among 13 objects, 7 objects lie on the first and third quadrants and 3 on second and fourth quadrants on the $\Delta$ EW versus $\Delta \log F(2500 \AA)$ diagram (their Figure 10), and the other three do not have significant variations in either parameter or both (less than their error bars). The situation is quite similar in the $\Delta \mathrm{EW}$ versus $\Delta \log F(1400 \AA)$ diagram: among 7 objects with significant variations of both parameters, 5 fall on the first and third quadrants and 2 on the second and fourth quadrants. The insignificance is simply due to the small number in the sample. Although Vivek et al. (2014) did not find any significant trend between the variations of absorption lines ( $\mathrm{Mg}$ II and $\mathrm{Al}$ III) and continuum flux, in their Table 3, six of eight quasars showed the opposite trend of long-term (on scales of years) continuum and absorption lines, only one displayed the same trend, and another one exhibited both weakening and strengthening BAL components. In this section, we will discuss the implication of these findings.

\subsection{What Drives Variations of Absorption Lines?}

Absorption-line variability can be caused by changes in either the gas ionization or the total gas column density. This can be produced by two main processes: changes in the ionizing continuum incident on the absorbing gas or gas moving in or out of the line of sight (Smith \& Penston 1988; Barlow et al. 1989; Barlow 1993). Previous studies did not reach a consensus on which one is the main driver. On the one hand, coordinated variations of different components of absorption lines suggest that the changes in different part of the outflows are driven by the same parameter. Most naturally, 

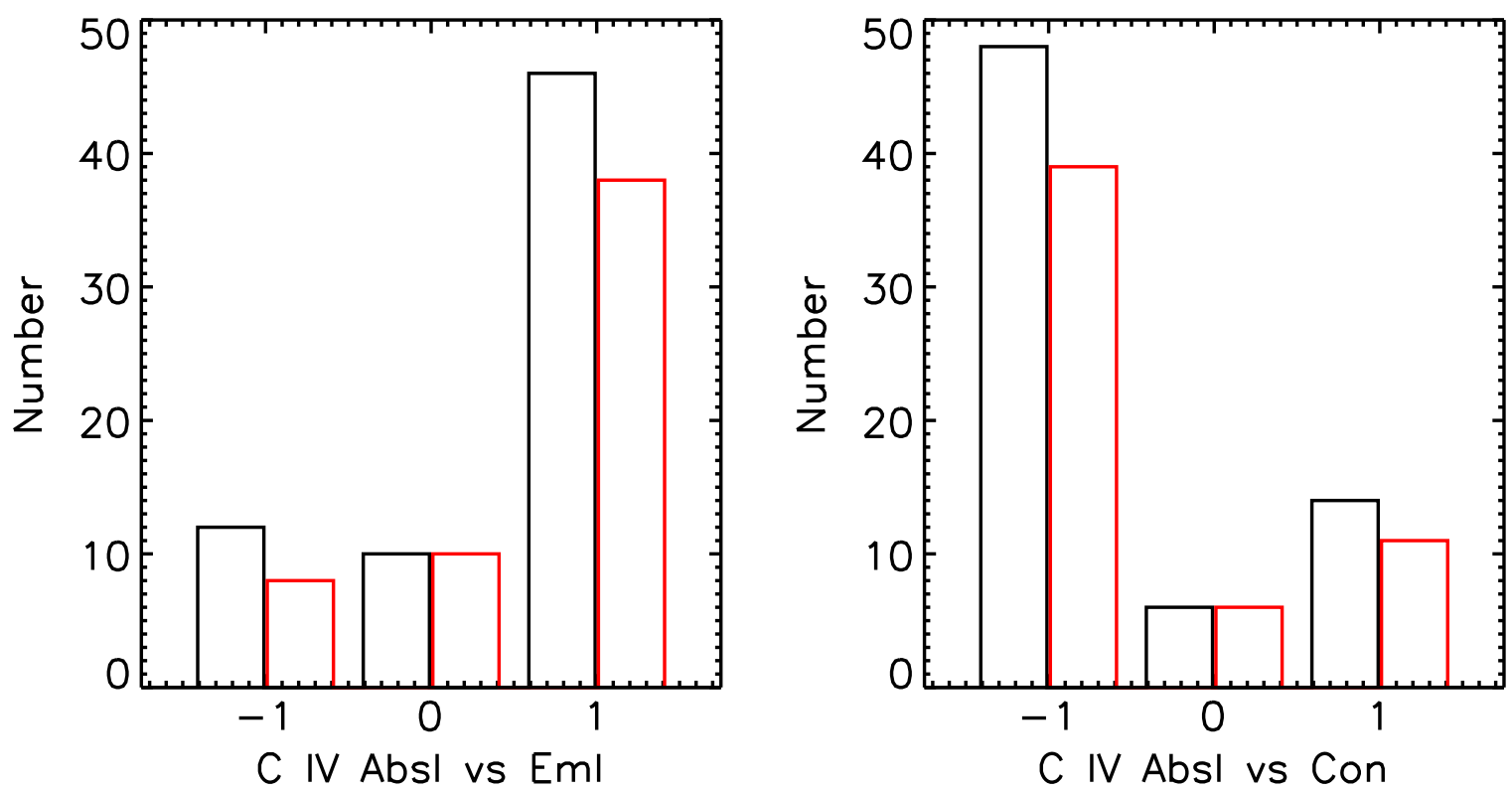

Figure 9. Distribution of concordance index between the $\mathrm{C}$ IV absorption line and continuum (right panel) and $\mathrm{C}$ iv absorption line vs. emission line (left panel) for quasars with one or more new BAL component. The black and red boxes are the same as in Figure 6.

this parameter is the ionizing continuum because it ionizes all gas producing these absorption components (Hamann et al. 2011; Capellupo et al. 2012). It is difficult to image the line-absorbing gas at different sites when moving in or out of the line of sight in step unless the flows are confined to a relatively small region. The latter is less likely, as components with large velocity differences will spread out on a relatively short timescale, but cannot be rejected completely. On the other hand, there are cases where a new C IV absorption-line component emerged or an old component disappeared. Such extreme variations can be readily explained as absorbing gas moves into or out of our line of sight (Hamann et al. 2008; Leighly et al. 2009; Krongold et al. 2010; Hall et al. 2011; Filiz Ak et al. 2013). Capellupo et al. (2014) argued that the similar variability trends of $\mathrm{Pv}, \mathrm{C}$ Iv, and $\mathrm{Si}$ IV in Q $1413+1143$ disfavored the continuum variations as the driver of $\mathrm{BAL}$ variability in this quasar.

Changes in the ionizing continuum striking the absorbing gas may be caused by the variations of the intrinsic ionizing continuum or/and by alteration of the column density or ionization of an intermediate absorber (refer to the discussion in Misawa et al. 2007; Filiz Ak et al. 2012, 2013), i.e., the shielding gas introduced to us by Murray et al. (1995). According to their model, shielding gas is optically thin to the observed UV continuum longward of $1000 \AA$. Thus, changes in the column density of shielding gas would not directly induce a correlated variation between UV continuum and absorption lines. On the other hand, if the main change in the ionization of shielding gas is caused by an increase or decrease of the ionization state in response to variations in the ionizing continuum, it will introduce a correlation between absorption lines and observed UV continuum. However, in this case, the ultimate driver of the absorption-line variability is the variation of the ionizing continuum.

The correlated variations between absorption lines and the continuum found in this paper directly support the idea that the intrinsic ionizing continuum is the main driver for absorptionline variability (see Barlow et al. 1992). We also showed that

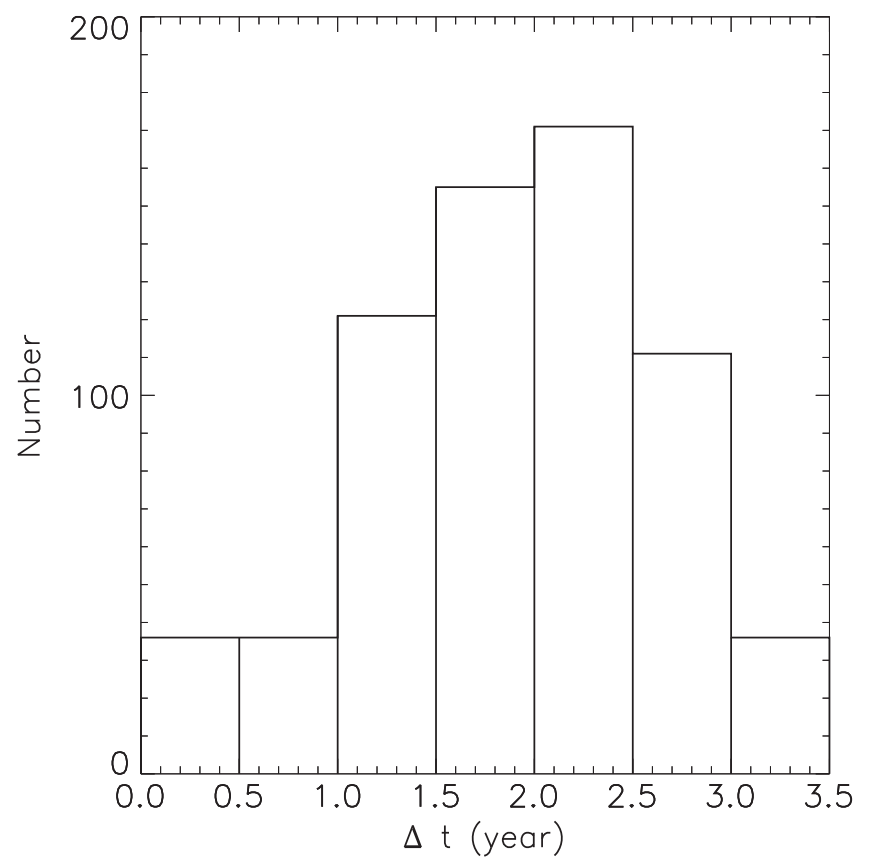

Figure 10. Distribution of time interval in the quasar rest frame between the two observations with the detection of absorption-line variations.

emergence or disappearance of an absorption-line component is closely correlated to the continuum variations, suggesting that they are caused by ionizing continuum variability as well. This indicates that there was/is persistently absorbing gas, but the ionization change is so large that the absorption line emerges/ disappears in the later epoch. We point out that large X-ray absorption column densities accompanying the transient BALs (Kaastra et al. 2014) cannot be simply explained by an ionization change, but rather are more likely due to the launching of a new outflow component or inserting of a new component of shielding gas. So, gas moving in and out may happen sometimes, but it is not the dominant mechanism driving quasar absorption-line variability. 

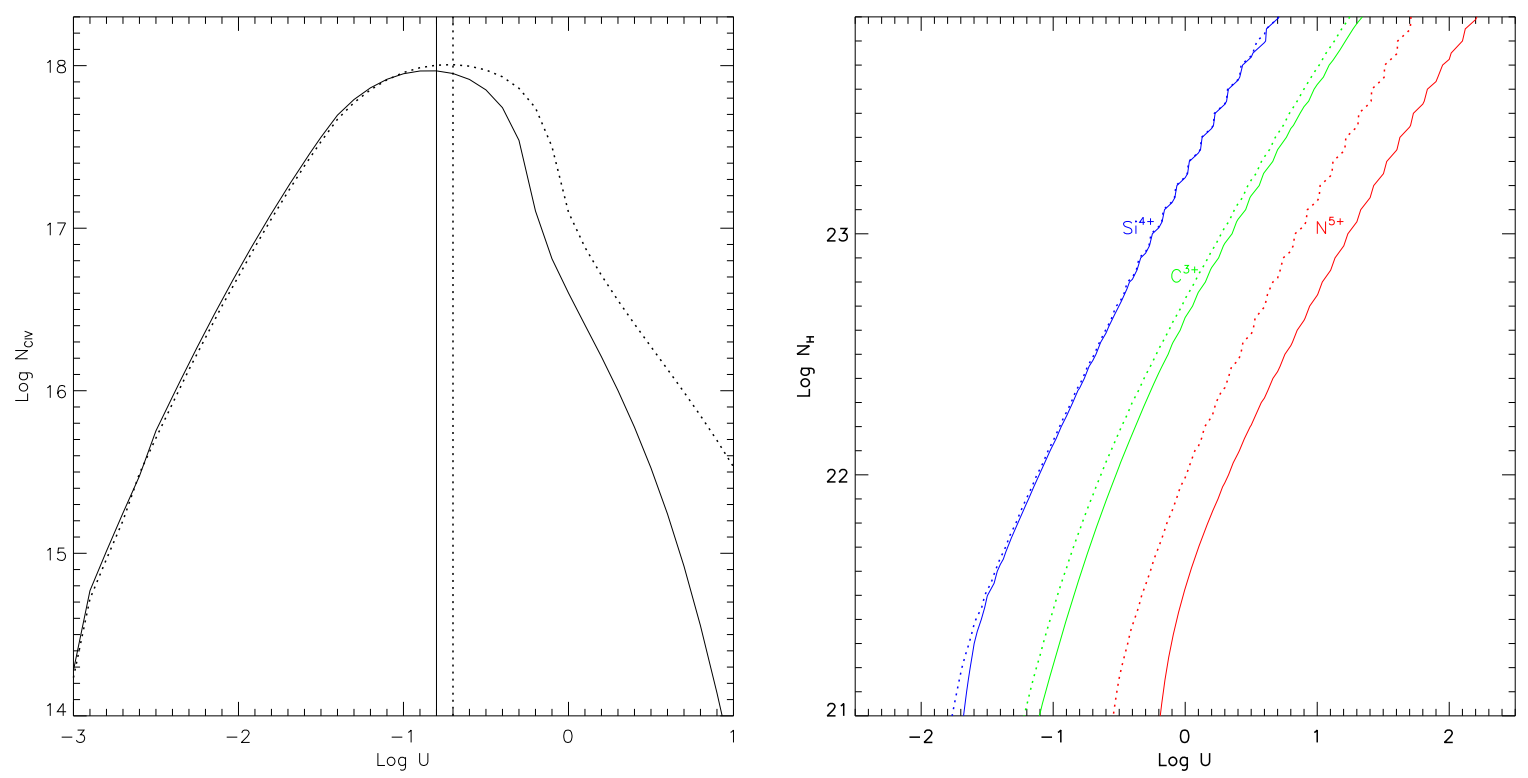

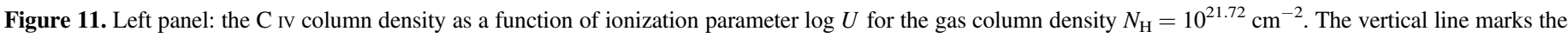

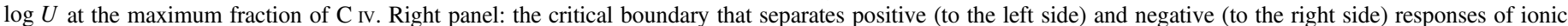

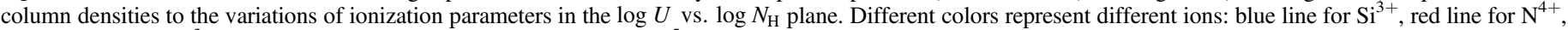

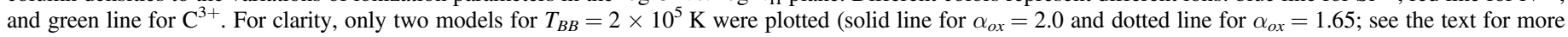
details).

\subsection{Implication for Concordant Continuum and Absorption- line Variations}

While highly concordant variations of absorption lines and the continuum strongly suggest that the response of gas ionization is to the ionizing continuum, it should be noted that this concordance is not an inevitable result of the latter. The ionic column density of a specific species may respond to a continuum variation positively or negatively, depending on the ionization of absorbing gas. This is illustrated in Figure 11, where the fraction of $\mathrm{C}^{3+}$ increases first, then reaches a peak, and decreases after as the ionization parameter increases. Moreover, if the outflow spans a large ionization range, one would expect that different parts may respond to the continuum variation differently. The coherence of different components indicates that the ionization of the outflows is globally higher or lower than the critical value that separates positive and negative responses. The fact that the depth of the absorption lines is inversely correlated with the continuum flux implies that the species more highly ionized than that producing the observed absorption lines always dominate. A further constraint can be drawn from the coherent variations of different absorption lines where $\mathrm{Si}$ Iv, $\mathrm{C}$ Iv, and $\mathrm{Nv}$ show the same variation sign. For instance, the gas ionization must be so high that most nitrogen is in ionization stages higher than $\mathrm{N}^{4+}$. Similarly, the statistical concordance of the variations of absorption lines and the continuum suggests that in most objects, outflows are highly ionized. To put further constraints on the ionization of the absorber, it would be interesting to check whether $\mathrm{O}$ VI or Ne VIII lines also vary in synchrony with other lines, which is beyond the scope of this paper. If this is indeed the case, these UV absorbers may be also responsible for the warm absorption observed in the X-ray band. The connection between X-ray and UV absorbers has been discussed for a long time, and it was proposed that at least some of the X-ray and UV absorbing material is physically connected in some Seyfert galaxies based on either a similar absorption-line profile or correlated temporal variations (e.g., Kaspi et al. 2002; Gabel et al. 2005; Kaastra et al. 2014), although the exact relation is not clear.

Alternatively, the outflow may be multi-phase with a range of ionization states, as recently proposed by Arav et al. (2013) based on an analysis of far-UV absorption lines of the quasar HE 0238-1904, or as is known in warm absorbers of Seyfert galaxies (e.g., Steenbrugge et al. 2009; Detmers et al. 2011). If the higher ionization phase dominates, then the competition between the positive response of ionic column density to the continuum variations in low ionization material and the negative response in high ionization gas may finally lead to the observed negative response in the BAL quasars. Detailed photoionization simulations are required to test whether this is a physically possible scenario and to constrain the physical parameter range.

The high concordance between continuum and absorptionline variations also requires a recombination time $t_{\text {rec }}=\left(n_{e} \alpha\right)^{-1}$ that is shorter than both the timescale of typical continuum variations and the interval between the two observations (e.g., Barlow et al. 1992). At a nominal temperature of 20,000 K (Hamann et al. 1997), the recombination rates for $\mathrm{C}_{\mathrm{IV}}$ to $\mathrm{C}_{\mathrm{III}}$ and $\mathrm{NV}_{\mathrm{V}}$ to $\mathrm{N}_{\mathrm{IV}}$ are $2.1 \times 10^{-11} \mathrm{~cm}^{3} \mathrm{~s}^{-1}$ and $2.6 \times 10^{-11} \mathrm{~cm}^{3} \mathrm{~s}^{-1}$, respectively (Badnell 2006) ${ }^{7}$. The ionization of a recombining gas will have a memory of the previous ionizing continuum for about a recombination timescale, i.e., the ionization of gas is connected with an average continuum over such a timescale. Continuum fluctuations on timescales shorter than this would not cause significant variations in absorption lines, rather, they would smear the correlation. Thus, we can use the interval between the two observations as an upper limit of the recombination time. The distribution of observational intervals for all pairs in

\footnotetext{
7 These recombination rates are about a factor of five larger than those of Arnaud \& Rothenflug (1985), adopted in the literature of most absorption-line studies.
} 


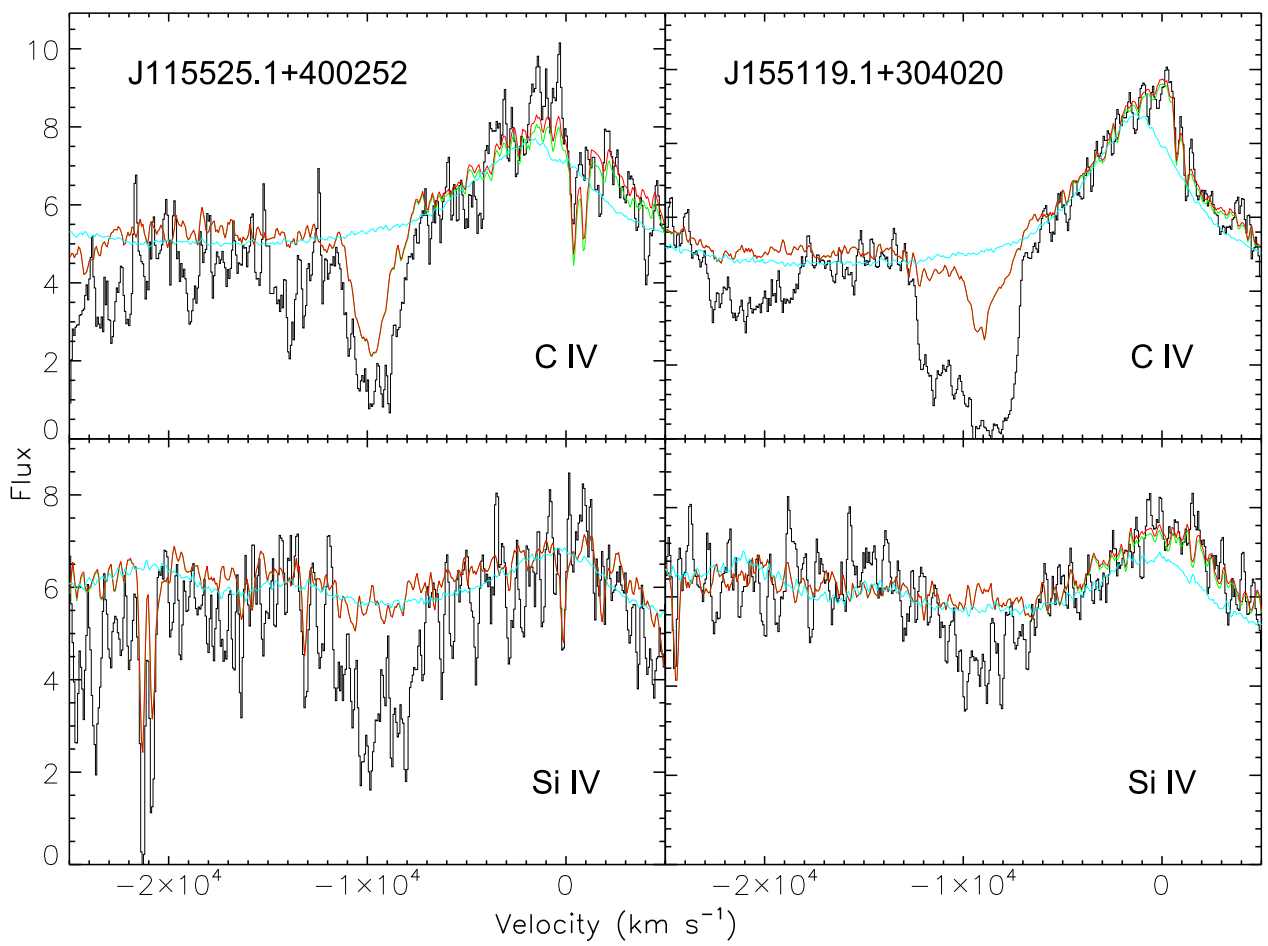

Figure 12. Two examples of the emergence or disappearance of Si Iv absorption lines. The SDSS spectra at one epoch and the scaled reference spectrum at another epoch are shown in black and green, respectively, as in Figure 4, while the unabsorbed QSO template is displayed in cyan. In the reference spectra (red), the C iv absorption line is prominent, while the Si IV absorption line is not significantly detected.

the sample is shown in Figure 10. Note that we do not count the minimum observed interval for significant absorption-line variation because we use the highest $\mathrm{S} / \mathrm{N}$ spectrum of an object as a reference.

Since absorption-line variability is caused by ionizing continuum variations, constraints on the gas density can be imposed using the absorption-line variability timescales (Hamann et al. 1997). Filiz Ak et al. (2013) showed that the fraction of BAL variations increases with an increasing time interval between the two observations, with the shortest detected changes in less than 10 days for $\mathrm{C}_{\mathrm{IV}}$ (see also Capellupo et al. 2013). With this timescale for C IV, one can set a lower limit on the electron density to be $n_{e} \geqslant\left(\alpha_{r} t\right)^{-1} \simeq(4-8) \times 10^{4} \mathrm{~cm}^{-3}$. For a typical active galactic nucleus (AGN) continuum and column density $>10^{22} \mathrm{~cm}^{-2}$, the ionization parameter should be larger than 1 (refer to Section 5.5), so the size of the absorption-line region should be less than a few tens of parsecs. It should be pointed out that the lack of variability on shorter timescales does not necessarily mean that we are detecting a minimum recombination/ionizing timescale. The power spectrum of AGN variability is rather red with a power law of slope of about -2.0 on timescales from days to several years (e.g., MacLeod et al. 2012), so the lack of short-timescale variability may be entirely attributed to very small variability amplitudes of the ionizing continuum on such a short timescale.

\subsection{On the Emergence and Disappearance of Absorption Lines}

If gas is dominated by higher ionization species, the emergence/disappearance of new $\mathrm{C}$ IV $\mathrm{BAL}$ troughs as the continuum weakens/brightening can be understood. The column density of a specific ion is initially too low to be detected, but when the ionizing continuum weakens, the species with the higher ionization recombines to raise the correspondent ions to a sufficient enough fraction to be detected. This actually has been observed in $\mathrm{Si}$ Iv lines associated with persistent but variable $\mathrm{C}_{\mathrm{IV}}$ absorption-line troughs. We found eight such cases in our sample, and two examples are shown in Figure 12. The emergence or disappearance of a C IV absorption-line component is an analog of such variations of Si IV, but it occurs at a higher ionization level. Large variations in the ionic column density can be attributed to either the large amplitude of the continuum variation or to the large response of the ionic column density to the continuum variations.

In order to show that large changes in the ionic column density can be produced by continuum variations, in the following, we explore analytically the physical parameter ranges that an ionic column density is very sensitive to the ionizing continuum variations from photoionization equilibrium analysis. We will use $\mathrm{C}^{3+}$ as an example. The equilibrium of $\mathrm{C}^{3+}$ is maintained by a balance between source terms, the recombination of $\mathrm{C}^{4+}$ plus photoionization of $\mathrm{C}^{2+}$, and sink terms, the recombination and photoionization of $\mathrm{C}^{3+}$ (Osterbrock \& Ferland 2006): ignoring three-body recombination at these low gas densities, we find

$$
\begin{gathered}
\text { source-sink }=0 \\
n_{e} n_{\mathrm{C}^{4+}} \alpha_{\mathrm{C}^{4+}}+n_{\mathrm{C}^{2+}} \int_{\nu_{\mathrm{C}^{2+}}}^{\infty} a_{\mathrm{C}^{2+}}(\nu) \frac{J(\nu)}{h \nu} d \nu-n_{\mathrm{C}^{3+}} \\
\times \int_{\nu_{\mathrm{C}^{3+}}}^{\infty} a_{\mathrm{C}^{3+}}(\nu) \frac{J(\nu)}{h \nu} d \nu-n_{e} n_{\mathrm{C}^{3+}} \alpha_{\mathrm{C}^{3+}}=0 .
\end{gathered}
$$

If carbon is dominated by $\mathrm{C}^{4+}$, the terms of recombination of $\mathrm{C}^{3+}$ and photoionization of $\mathrm{C}^{2+}$ will be relatively small in comparison with the other two terms. So we can rewrite the 
equation approximately as

$$
n_{e} n_{\mathrm{C}^{4+}} \alpha_{\mathrm{C}^{4+}} \simeq n_{\mathrm{C}^{3+}} \int_{\nu_{\mathrm{C}^{3+}}}^{\infty} a_{\mathrm{C}^{3+}}(\nu) \frac{J(\nu)}{h \nu} d \nu
$$

If the shape of the ionizing continuum remains the same (in the optically thin case) and if the ionization is dominated by higher ionization species, then the number ratio of $\mathrm{C}^{3+}$ to $\mathrm{C}^{4+}$ can be written approximately with

$$
\frac{n_{\mathrm{C}^{3+}}}{n_{\mathrm{C}^{4+}}} \propto U^{-1} \text {. }
$$

If $\mathrm{C}^{5+}$ is the dominant species, then $n_{\mathrm{C}^{3+}} \propto U^{-2}$. On the other hand, if $\mathrm{C}^{6+}$ is the dominant one, $n_{\mathrm{C}^{3+}} \propto U^{-3}$. A similar analysis can be carried out for other ions, such as $\mathrm{Si}^{3+}$ and $\mathrm{N}^{4+}$. For an optically thick gas, the rapid depletion of higher ionization species near the ionization front would make the slope even steeper than in the above optically thin case. Photoionization model calculations (refer to Section 5.5) suggest an even steeper slope that can reach -5 for Si IV, $\mathrm{C}$ IV, and $\mathrm{N} v$ for some extreme parameters. Thus, the column density variations can be large even if the continuum variation is only modest, provided that the ionization is high and the total column density is sufficiently large. One should note that if there is shielding gas, the situation would occur because shielding gas is more transparent to the ionizing continuum at higher photon energies, leading to the ionizing continuum softening as the continuum is extinguished.

If above interpretation is correct, an interesting implication is that there are persistent high ionization outflows, which may not be detectable in the UV. But when the continuum weakens considerably, the gas ionization lowers to a level so that an appreciable fraction of ions are in the correct ionization state, so one can detect UV absorption lines. These outflows may already have been detected in X-ray spectra. Gallagher et al. (2005) detected strong X-ray absorption (column density of $\leqslant 10^{22} \mathrm{~cm}^{-2}$ ) in quasars with large $\mathrm{C}$ IV blueshifted emission lines but without absorption lines, and they suspected that the X-ray absorbing gas is highly ionized. Such highly ionized outflows may be an analog to the so-called ultrafast outflows (UFOs) as observed in the X-ray spectra of Seyfert galaxies (Tombesi et al. 2012). Note that the typical velocity of UFOs is $0.1 c$, which overlaps with BAL outflows, as do their column densities (Hamann et al. 2013).

\subsection{Photoionization Models}

In order to see how ionic column densities change with changes in the ionizing continuum, we run a series of photoionization simulations using version c13.03 of Cloudy, last described by Ferland et al. (2013). We consider a typical gas density of $10^{6} \mathrm{~cm}^{-3}$ since gas ionization is not sensitive to density at a given ionization parameter $U$. In practice, we compute models in $-3 \leqslant \log U \leqslant 3$ with a step $\Delta \log U=0.1, \quad$ and $20 \leqslant \log N_{\mathrm{H}} \leqslant 24$ with a step $\Delta \log N_{\mathrm{H}}=0.2$. Previous observations showed that BAL gas is optically thin to the ionizing photons (e.g., Lu et al. 2008; Baskin et al. 2013), suggesting that there is no hydrogen ionization front within outflows. Thus, we need only to consider a fraction of the parameter space on the $\log U$ versus $\log N_{\mathrm{H}}$ diagram, which runs diagonally in the $\log U$ versus $\log N_{\mathrm{H}}$ diagram.
We use the following form of the SED (Mushotzky \& Ferland 1984) to describe the ionizing continuum shape:

$$
f_{\nu}=A \nu^{-\alpha_{\mathrm{UV}}} \exp \left(-h \nu / k T_{B B}\right)+B \nu^{-\alpha_{X}},
$$

where the first term represents the big blue bump in the UV and the second term the power-law X-ray continuum. The parameter $k T_{B B}$ describes the cutoff energy of thermal emission from the accretion disk, while $\alpha_{o x}=-\log \left(f_{2 \mathrm{keV}} / f_{2500 \AA}\right) / \log \left(\nu_{2 \mathrm{keV}} / \nu_{2500 \AA}\right) \quad$ characterizes the relative contribution of the power law in the X-ray band, i.e., related to the ratio of $A$ and $B$. There are still large uncertainties in the ionizing continuum shape of quasars in the far-UV band. It is inaccessible for low-redshift quasars due to the Galactic interstellar medium, and there are large uncertainties in the intergalactic absorption corrections for high-redshift quasars. Therefore, we adopt the above empirical description rather than Hubble Space Telescope or FUSE composite spectrum of quasars (Zheng et al. 1997; Stevans et al. 2014). We choose two different $k T_{B B}=1.5 \times 10^{5} \mathrm{~K}$ and $2 \times 10^{5} \mathrm{~K}$ to examine the effect of the far-UV continuum and two $\alpha_{o x}=-2.0,-1.65$, representing the effect of our ignorance of the intrinsic X-ray strength for these quasars (Gallagher et al. 2006; Fan et al. 2009) on the final results. The slope $\alpha_{\mathrm{UV}}$ is held fixed at -0.5 .

It is worth noting that if there is shielding gas, to prevent the outflow being overionized so to keep line driving acceleration effective, the ionizing continuum incident on the outflow will be more complicated. While large X-ray absorption (equivalent to cold absorption of column density $N_{\mathrm{H}} \sim 10^{22}-10^{24} \mathrm{~cm}^{-2}$ ) is usually detected in the X-ray spectrum of BAL QSOs, whether it is highly ionized or not remains uncertain due to the weakness of their X-ray emission (Gallagher et al. 2006; Fan et al. 2009). It is also controversial whether the X-ray absorber is the same material as the UV absorber (Hamann 1998; Mathur et al. 1998; Wang et al. 2000) or is the shielding gas at the base of the outflow. Compton thick X-ray absorption has been reported for some low ionization BAL QSOs based on the weakness of X-ray emission (e.g., Morabito et al. 2011). Because the UV continuum will be subject to very large attenuation due to electron scattering, the intrinsic UV luminosity would need to be too high to be correct. However, the conclusion is based on the assumption that BAL QSOs have a similar intrinsic X-ray to the optical luminosity ratio, which need not be true (Fan et al. 2009; Morabito et al. 2014). In addition, there is no X-ray absorption in high-velocity miniBAL QSOs, suggesting that such shielding gas is not necessary for an outflow to be accelerated (Hamann et al. 2013). In fact, if outflows have the high ionization parameters discussed here, it is plausible that the X-ray absorption observed in the BAL quasars can be formed in the outflow itself. The recent emergence of both X-ray absorption and UV absorption lines in the Seyfert galaxies Mrk 335 and NGC 5548 seems to support this scenario (Longinotti et al. 2013; Kaastra et al. 2014). Future simultaneous observations of transient BAL events in X-ray and UV can test this.

Only equilibrium models are considered, i.e., we assume that continuum variations are slower than the gas recombination or photoionization timescales. This is likely a fair approximation because the ion column density change traces fairly well the continuum variations. The requirement that the recombination time of $\mathrm{C}$ IV, $\mathrm{Nv}$, and $\mathrm{Si}$ IV be shorter than than the shortest 
variability timescale explored in this paper $(\sim 0.1$ years) converts to a density of $n>2 \times 10^{4} \mathrm{~cm}^{-3}$ (see Section 5.3). Non-equilibrium ionization will require time-dependent photoionization models and knowledge of the continuum light curve. It is beyond the scope of this paper, but may be necessary in interpreting high-cadence spectroscopic monitoring data.

We show the parameter regimes where $\mathrm{C}$ IV, $\mathrm{Si}$ IV, and $\mathrm{N} v$ respond negatively to an increasing ionization parameter in Figure 11. They are located on the lower right side of the critical curves in the figure. As expected, the critical curves of $\mathrm{Si}$ IV and $\mathrm{C}$ IV do not depend much on the ionizing continuum shape, while that of $\mathrm{NV}$ changes substantially with the two SEDs adopted here. That is because the two SEDs result in a large difference in the $\mathrm{Nv}$ ionizing photons at a given $\log U$. Apparently, our results suggest that the ionization of the gas is high and most populated species of nitrogen and carbon are in an ionization stage higher than Li-like ions. A critical examination of variability of higher ionization species such as $\mathrm{O}$ vi and Ne VIII would be important to constrain how high the ionization of gas might be. We also show the contours of the response of ion column densities to variations of the ionization parameter $(d \log N / d \log U)$ in Figure 13. Apparently, at large ionization parameters and large column densities, the response is very large, with a slope as steep as -5 for $\mathrm{C}$ IV, $\mathrm{Nv}$, and Si IV in some zones of the diagram. One interesting feature in the figure is that $\mathrm{C}$ IV and $\mathrm{Nv}$ have an additional narrow diagonal stripe zone of a slope $\leqslant-5$ to the left such border of the Si Iv, which is especially obvious when X-rays are weak. We have checked and find that these regions correspond to the formation of an ionization front of $\mathrm{C} \mathrm{V}$ or $\mathrm{N} \mathrm{VI}$, on which the ion column density is very sensitive to change in ionization parameter. In these parameter regimes, a relatively small variation in the continuum luminosity may cause a large change in the optical depth of the absorption line, so only moderate continuum variations are required to explain the observed disappearance or emergence of an absorption-line component for a specific ion. The regimes are highly overlapped for $\mathrm{N} v$ and $\mathrm{C}$ IV in the higher ionization parameter zone, suggesting that the emergence or disappearance of $\mathrm{N} \mathrm{V}$ and $\mathrm{C}$ IV may occur at the same time. A thorough analysis of this will be given in the future after properly modeling the spectrophotometric calibration uncertainty.

\section{CONCLUSION}

We analyze the variability of BALs and mini-BALs and their correlations with those of the continuum and emission lines for a sample of 452 quasars in the redshift range $2.2<z \leqslant 4.7$ and with multi-epoch SDSS spectroscopic observations. Variations among different components of $\mathrm{C}$ IV, $\mathrm{N} \mathrm{v}$, and $\mathrm{Si}$ IV absorption lines, or the same component of different lines, are highly coordinated. These conclusions are consistent with previous studies of C IV, Si IV, and extend to Nv (Hamann et al. 2011; Capellupo et al. 2012; Filiz Ak et al. 2013). We find that variations of these absorption lines are also highly synchronized with those of the continuum and emission lines. The absorption lines weaken/strengthen statistically when the continuum brightens/dims. The uncertainties in the continuum flux calibration prevent us from assessing the detailed fraction of quasar absorption lines where the absorption-line variability does not follow continuum variations. We also found 50 cases of the emergence or disappearance of an absorption-line component that accompanies changes in the continuum. We interpret these results as indicating that variations in the ionizing continuum are the main driver for the absorption-line variability and that the dominate species are ions higher than the observed Li-like ions for carbon and nitrogen. For a reasonable ionizing continuum and gas column density, these constraints imply an ionization parameter of $\log U \geqslant 0$. In the case of the disappearance and emergence of BAL components, the ionization parameters should be even higher if continuum luminosity does not change by a large factor during the period, indicating the presence of persistent highly ionized outflows even when UV absorption lines have disappeared. This can be further tested with future X-ray observations. We cannot rule out the possibility that gas moving in or out of the line of sight may account for the variation of BALs, including their emergence and disappearance, in some quasars.

We thank the referee for useful comments. We acknowledge the financial support by the Strategic Priority Research Program "The Emergence of Cosmological Structures" of the Chinese Academy of Sciences (XDB09000000), NSFC through (NSFC-11233002, NSFC-11421303, U1431229) and National Basic Research Program of China (grant No. 2015CB857005). G.J.F. is grateful to the Leverhulme Trust for support via the award of a Visiting Professorship at Queens University Belfast (VP1-2012-025). G.J.F. acknowledges support by NSF (1108928, 1109061, and 1412155), NASA (10-ATP10-0053, 10-ADAP10-0073, NNX12AH73G, and ATP13-0153), and STScI (HST-AR-13245, GO-12560, HSTGO-12309, GO-13310.002-A, and HST-AR-13914). Funding for SDSS-III has been provided by the Alfred P. Sloan Foundation, the Participating Institutions, the National Science Foundation, and the U.S. Department of Energy Office of Science. The SDSS-III website is http://www.sdss3.org/. SDSS-III is managed by the Astrophysical Research Consortium for the Participating Institutions of the SDSS-III Collaboration.

\section{APPENDIX \\ EFFECTS OF SPECTROPHOTOMETRIC CALIBRATION UNCERTAINTIES ON THE SIGN OF CONTINUUM VARIATIONS OF VARIABLE BAL QSOs}

The fiber positions of BOSS quasar targets were purposefully offset in order to optimize the throughput of light at $4000 \AA$, while the standard stars used for flux calibration are positioned for $5400 \AA$. This results in a large uncertainty in the flux calibration of quasar spectra in the BOSS survey (Dawson et al. 2013). In this Appendix, we will estimate the effect of spectrophotometric calibration uncertainties on the statistical properties of the sign of the continuum variation defined in Section 3 using stars that were accidentally observed as quasars in the SDSS legacy and SDSS BOSS and $r<20.5$. We assume these stellar spectra have similar calibration uncertainties to the spectra of BOSS quasar targets (See Dawson et al. 2013).

We extract the photometric and spectroscopic synthetic magnitudes of these stars from the SDSS DR10 database. The query resulted in 98,079 stars. We remove M stars, which usually have faint magnitudes and very red colors, and so differ from those of most quasars in the sample. This leaves a sample of 79,237 stars, including 37,487 from the SDSS legacy program. We calculate the differences between photometric and spectrosynthesis magnitudes for stars observed in the legacy and BOSS programs. The difference is caused by a 


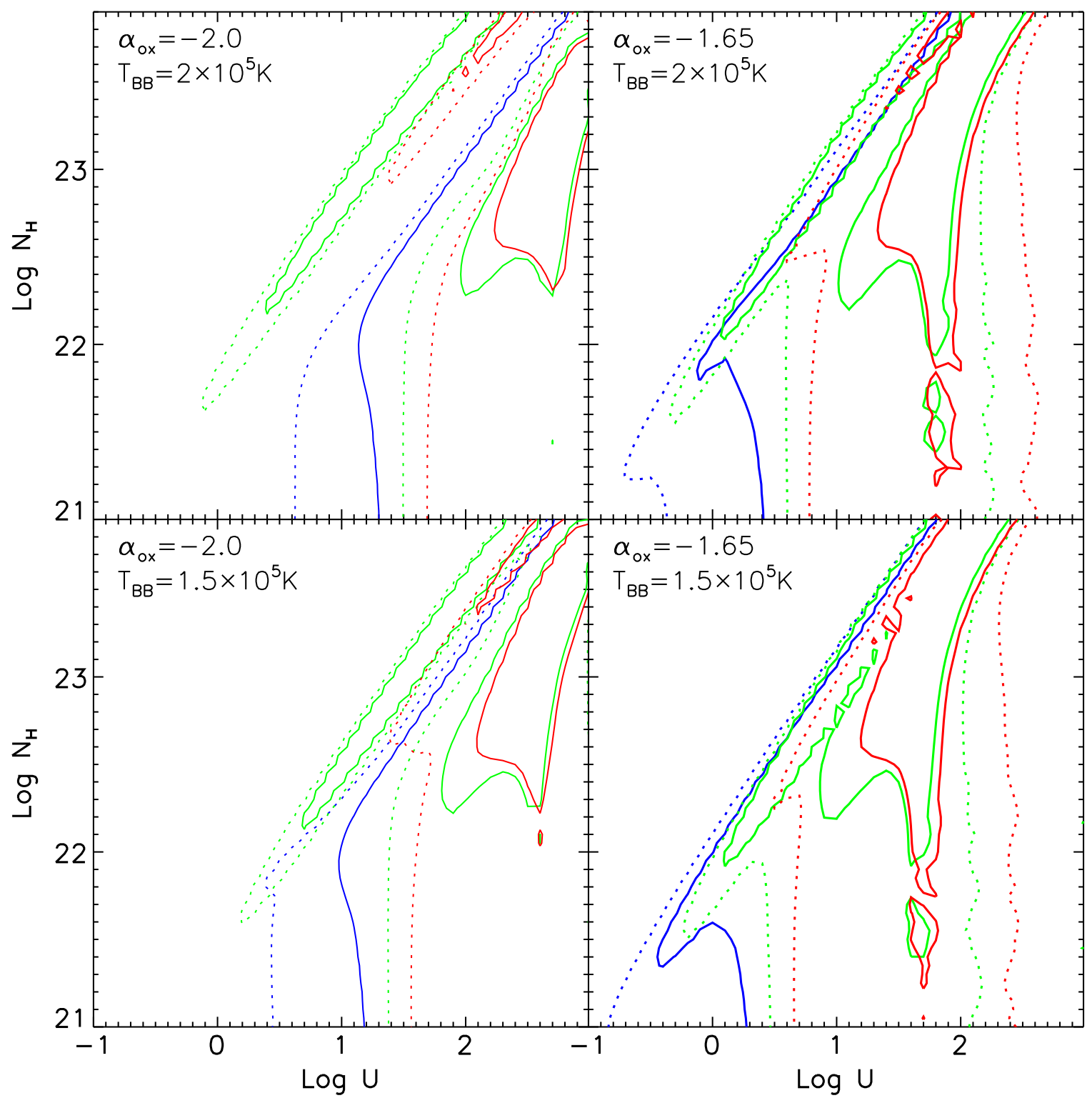

Figure 13. Contour for large negative response of $\mathrm{Si}^{3+}$ (blue), $\mathrm{N}^{4+}$ (red), and $\mathrm{C}^{3+}$ (green). The dashed line is for $d \log N / d \log U=-3$, and the solid line is for $d \log N / d \log U=-5$. The shape parameters of the ionizing continuum are marked in the left corner of each panel. For $\alpha_{o x}=-1.65$ at $\log U>2$, the gas is too highly ionized to produce a significant response for either $\mathrm{C}$ IV or $\mathrm{N}$ IV.

combination of photon noise, imperfect background subtraction, possible variability of some of these stars, and the systematic flux calibration uncertainty. The first two terms are relatively small in comparison to the observed difference because the typical uncertainty in magnitude given by the SDSS pipeline is 0.029 mag in $g$. Most stars should be stable at a level of $1 \%$. Neglecting the first three terms, we attribute the difference mostly to the flux calibration error, and thus give a conservative estimate of the latter.

We plot the distributions of the difference magnitude (DDMs) in the $g$-band $\left(g-g_{\text {synth }}\right)$ for stars observed in the BOSS and legacy programs separately in Figure 14. There is a small $(-0.05 \mathrm{mag})$ systematic offset in the peak of the distribution for the BOSS spectra, but no such offset in the legacy spectra. In either case, the distribution is not symmetric, but rather is skewed to the negative, i.e., a fraction of stars appear fainter in the SDSS spectrum than they should be. Also the difference distribution for the BOSS spectra is much broader than that for the legacy spectra, suggesting large calibration errors. When splitting stars into different spectroscopic types, we see similar distributions for types B, A, G, F, and $\mathrm{K}$. Type $\mathrm{O}$ stars have a significantly broader wing than other types for unknown reasons. Because of this, we do not try to do any color-related corrections. We also checked the distributions in other bands and found that they are significantly broader than in the $g$-band. Therefore, we will mainly use the $g$-band. This approximately corresponds to a rest-frame wavelength of $1400 \AA$ for the majority of our quasars.

Next, we estimate the approximate average error distribution of the differential synthetic magnitudes of quasar spectra obtained at two different epochs (DDSM for short) using the DDMs described above. We convolve the DDM of one spectrum with that of another assuming that their errors are uncorrelated to obtain the DDSM of a specific spectrum pair. Since the spectra taken in legacy and BOSS have different 


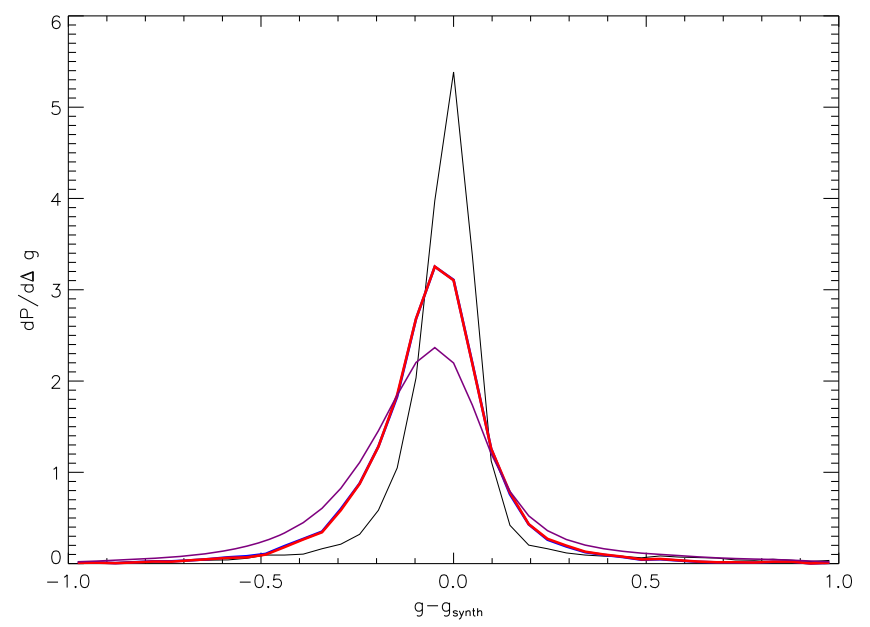

Figure 14. Distribution of difference magnitudes in the photometric psf magnitude and the spectroscopic synthetic magnitudes for stars mistargeted as quasars in the SDSS legacy and BOSS spectroscopic survey programs. Spectroscopic targets in the legacy program are plotted as a black line, while those in the BOSS program are plotted as a blue line. For comparison, the average two-epoch magnitude difference spread function (see the text for details) for a variable BAL QSO sample is also shown as a red line.

DDMs, we obtain four different DDSMs for combinations: (legacy, legacy), (legacy, BOSS), (BOSS, legacy), and (BOSS, BOSS). The systematic offset is subtracted from the DDM for the BOSS spectra. We take an average of the DDSM (Figure 14), weighted with the number of quasar pairs of our variable BAL quasars in each combination. The distribution is fairly broad, suggesting that it is impossible to quantitatively estimate the variations of an individual spectrum pair. Note that this treatment is only a conservative approximation since quasars in ancillary programs may not be observed in the mode of fiber position offset, and it was shown that the flux calibration error is smaller in the latter case (Dawson et al. 2013).

Finally, we calculate the two-epoch magnitude difference (TEMD) for quasar pairs in Table 1 by correcting the systematic offset in the spectrophotometric calibration in the BOSS survey for quasars. The difference magnitude at $1400 \AA$ is calculated by using the double power-law scaling factor (Equation (1)) in the reference-matching procedure (Section 2.2). The distribution of TEMD (Figure 15) is considerably broader than the average DDSM, suggesting that variability information can be extracted statistically. The distribution cannot be fitted by a single-Gaussian function convolved with DDSM, so we fit it with the sum of two Gaussian functions. Figure 15 shows the best fit and the double-Gaussian model for the intrinsic distribution. We take the latter as the true distribution for magnitude variations and examine what fraction of sources were assigned to a wrong sign of variations using the definition in this paper after convolving the positive and negative part of the distribution with the two-epoch MSF. We find that $13 \%$ of sources were assigned opposite signs due to the calibration uncertainties, and $10 \%$ were assigned uncertain signs, i.e., magnitude difference between -0.05 and $0.05 \mathrm{mag}$. Considering that some quasars in the ancillary programs were not observed in an offset mode, we likely overestimate the number of pairs with misassigned signs.

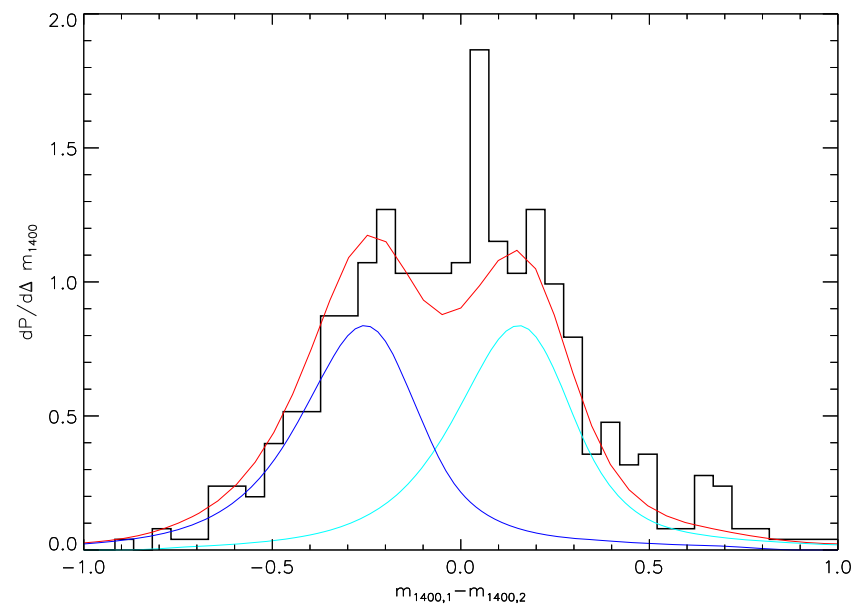

Figure 15. Distribution of the difference of spectral synthetic magnitudes at two epochs of the variable BAL QSO sample. The red line represents the bestfitted double-Gaussian model convolved with the two- epoch magnitude difference spread function in Figure 14. The blue and cyan lines are the convolved positive and negative parts of the intrinsic distributions, respectively.

\section{REFERENCES}

Abazajian, K. N., Adelman-McCarthy, J. K., Agüeros, M. A., et al. 2009, ApJS, 182, 543

Arav, N., Borguet, B., Chamberlain, C., Edmonds, D., \& Danforth, C. 2013, MNRAS, 436, 3286

Arnaud, M., \& Rothenflug, R. 1985, A\&AS, 60, 425

Badnell, N. R. 2006, ApJS, 167, 334

Barlow, T. A. 1993, PhD thesis, Univ. of California, San Diego

Barlow, T. A., Hamann, F., \& Sargent, W. L. W. 1997, in ASP Conf. Ser. Vol. 128, Mass Ejection from Active Galactic Nuclei, ed. N. Aravm, I. Shlosman, \& R. J. Weymann (San Francisco, CA: ASP), 13

Barlow, T. A., Junkkarinen, V. T., \& Burbidge, E. M. 1989, ApJ, 347, 674

Barlow, T. A., Junkkarinen, V. T., Burbidge, E. M., et al. 1992, ApJ, 397, 81

Barlow, T. A., \& Sargent, W. L. W. 1997, AJ, 113, 136

Baskin, A., Laor, A., \& Hamann, F. 2013, MNRAS, 432, 1525

Borguet, B. C. J., Arav, N., Edmonds, D., Chamberlain, C., \& Benn, C. 2013, ApJ, 762, 49

Capellupo, D. M., Hamann, F., \& Barlow, T. A. 2014, MNRAS, 444, 1893

Capellupo, D. M., Hamann, F., Shields, J. C., Halpern, J. P., \& Barlow, T. A. 2013, MNRAS, 429, 1872

Capellupo, D. M., Hamann, F., Shields, J. C., Rodríguez Hidalgo, P., \& Barlow, T. A. 2011, MNRAS, 413, 908

Capellupo, D. M., Hamann, F., Shields, J. C., Rodríguez Hidalgo, P., \& Barlow, T. A. 2012, MNRAS, 422, 3249

Chartas, G., Saez, C., Brandt, W. N., Giustini, M., \& Garmire, G. P. 2009, ApJ, 706, 644

Corbin, M. R., \& Boroson, T. A. 1996, ApJS, 107, 69

Dawson, K. S., Schlegel, D. J., Ahn, C. P., et al. 2013, AJ, 145, 10

Detmers, R. G., Kaastra, J. S., Steenbrugge, K. C., et al. 2011, A\&A, 534, A38

Fan, L. L., Wang, H. Y., Wang, T., et al. 2009, ApJ, 690, 1006

Ferland, G. J., Porter, R. L., van Hoof, P. A. M., et al. 2013, RMxAA, 49, 137

Filiz Ak, N., Brandt, W. N., Hall, P. B., et al. 2012, ApJ, 757, 114

Filiz Ak, N., Brandt, W. N., Hall, P. B., et al. 2013, ApJ, 777, 168

Foltz, C. B., Weymann, R. J., Morris, S. L., \& Turnshek, D. A. 1987, ApJ, 317,450

Gabel, J. R., Kraemer, S. B., Crenshaw, D. M., et al. 2005, ApJ, 631, 741

Gibson, R. R., Brandt, W. N., Gallagher, S. C., Hewett, P. C., \& Schneider, D. P. 2010, ApJ, 713, 220

Gibson, R. R., Brandt, W. N., Gallagher, S. C., \& Schneider, D. P. 2009, ApJ, 696, 924

Gibson, R. R., Brandt, W. N., Schneider, D. P., \& Gallagher, S. C. 2008, ApJ, 675,985

Gallagher, S. C., Brandt, W. N., Chartas, G., et al. 2006, ApJ, 644, 709

Gallagher, S. C., Richards, G. T., Hall, P. B., et al. 2005, AJ, 129, 567

Ganguly, R., \& Brotherton, M. S. 2008, ApJ, 672, 102

Hall, P. B., Anosov, K., White, R. L., et al. 2011, MNRAS, 411, 2653

Hall, P. B., Brandt, W. N., Petitjean, P., et al. 2013, MNRAS, 434, 222

Hamann, F. 1998, ApJ, 500, 798 
Hamann, F., Barlow, T. A., \& Junkkarinen, V. 1997, ApJ, 478, 87 Hamann, F., Chartas, G., McGraw, S., et al. 2013, MNRAS, 435, 133

Hamann, F., Kanekar, N., Prochaska, J. X., et al. 2011, MNRAS, 410, 1957

Hamann, F., Kaplan, K. F., Rodríguez Hidalgo, P., Prochaska, J. X., \& Herbert-Fort, S. 2008, MNRAS, 391, L39

Hamann, F., \& Sabra, B. 2004, in ASP Conf. Ser. Vol. 311, AGN Physics with the Sloan Digital Sky Survey, ed. G. T. Richards \& P. B. Hall (San Francisco, CA: ASP), 203

Kaastra, J. S., Kriss, G. A., Cappi, M., et al. 2014, Sci, 345, 64

Kaspi, S., Brandt, W. N., George, I. M., et al. 2002, ApJ, 574, 643

Kinney, A., Rivolo, A. R., \& Koratkar, A. P. 1990, ApJ, 357, 338

Korista, K. T., Bautista, M. A., Arav, N., et al. 2008, ApJ, 688, 108

Kormendy, J., \& Ho, L. C. 2013, ARA\&A, 51, 511

Krongold, Y., Binette, L., \& Hernández-Ibarra, F. 2010, ApJL, 724, L203

Leighly, K. M., Hamann, F., Casebeer, D. A., \& Grupe, D. 2009, ApJ, 701, 176

Liu, W.-J., Zhou, H., Ji, T., et al. 2015, ApJS, 217, 11

Longinotti, A. L., Krongold, Y., Kriss, G. A., et al. 2013, ApJ, 766, 104

Lu, H., Wang, T., Yuan, W., et al. 2008, ApJ, 680, 858

Lundgren, B. F., Wilhite, B. C., Brunner, R. J., et al. 2007, ApJ, 656, 73

MacLeod, C. L., Ivezić, Ž., Sesar, B., et al. 2012, ApJ, 753, 106

Mathur, S., Wilkes, B., \& Elvis, M. 1998, ApJL, 503, L23

Misawa, T., Eracleous, M., Charlton, J. C., \& Kashikawa, N. 2007, ApJ, 660,152

Morabito, L. K., Dai, X., Leighly, K. M., Sivakoff, G. R., \& Shankar, F. 2011 ApJ, 737, 46

Morabito, L. K., Dai, X., Leighly, K. M., Sivakoff, G. R., \& Shankar, F. 2014, ApJ, 786, 58

Murray, N., Chiang, J., Grossman, S. A., \& Voit, G. M. 1995, ApJ, 451, 498

Mushotzky, R., \& Ferland, G. J. 1984, ApJ, 278, 558

Osterbrock, D. E., \& Ferland, G. J. 2006, in Astrophysics of Gaseous Nebulae and Active Galactic Nuclei, ed. D. E. Osterbrock \& G. J. Ferland (2nd ed.; Sausalito, CA: Univ. Science Books), 50
Pâris, I., Petitjean, P., Aubourg, É., et al. 2014, A\&A, 563, A54

Richards, G. T., Hall, P. B., Vanden Berk, D. E., et al. 2003, AJ, 126, 1131

Richards, G. T., Kruczek, N. E., Gallagher, S. C., et al. 2011, AJ, 141, 167

Rodríguez Hidalgo, P., Eracleous, M., Charlton, J., et al. 2013, ApJ, 775 14

Schneider, D. P., Richards, G. T., Hall, P. B., et al. 2010, AJ, 139, 2360

Shen, Y., Richards, G. T., Strauss, M. A., et al. 2011, ApJS, 194, 45

Silk, J. 2011, in IAU Symp. 277, Tracing the Ancestry of Galaxies, ed C. Carignan, F. Combes, \& K. C. Freeman (Cambridge: Cambridge Univ. Press), 273

Smith, L. J., \& Penston, M. V. 1988, MNRAS, 235, 551

Steenbrugge, K. C., Fenovčík, M., Kaastra, J. S., Costantini, E., \& Verbunt, F. 2009, A\&A, 496, 107

Stevans, M. L., Shull, J. M., Danforth, C. W., \& Tilton, E. M. 2014, ApJ, 794, 75

Tombesi, F., Cappi, M., Reeves, J. N., \& Braito, V. 2012, MNRAS, 422, L1

Tombesi, F., Meléndez, M., Veilleux, S., et al. 2015, Natur, 519, 436

Trump, J. R., Hall, P. B., Reichard, T. A., et al. 2006, ApJS, 165, 1

Turnshek, D. A., Grillmair, C. J., Foltz, C. B., \& Weymann, R. J. 1988, ApJ, 325,651

Vivek, M., Srianand, R., Petitjean, P., et al. 2014, MNRAS, 440, 799

Wang, T. G., Brinkmann, W., Yuan, W., Wang, J. X., \& Zhou, Y. Y. 2000, ApJ, 545, 77

Wang, H., Wang, T., Zhou, H., et al. 2011, ApJ, 738, 85

Wang, H., Zhou, H., Yuan, W., \& Wang, T. 2012, ApJL, 751, L23

Welling, C. A., Miller, B. P., Brandt, W. N., Capellupo, D. M., \& Gibson, R. R. 2014, MNRAS, 440, 2474

Weymann, R. J., Morris, S. L., Foltz, C. B., \& Hewett, P. C. 1991, ApJ, 373,23

Wildy, C., Goad, M. R., \& Allen, J. T. 2014, MNRAS, 437, 1976

Zhang, S., Wang, H., Wang, T., et al. 2014, ApJ, 786, 42

Zheng, W., Kriss, G. A., Telfer, R. C., Grimes, J. P., \& Davidsen, A. F. 1997, ApJ, 475, 469 